MATHEMATICS OF COMPUTATION

Volume 67, Number 224, October 1998, Pages 1361-1382

S 0025-5718(98)00993-4

\title{
AN ADAPTIVE STRATEGY \\ FOR ELLIPTIC PROBLEMS INCLUDING A POSTERIORI CONTROLLED BOUNDARY APPROXIMATION
}

\author{
W. DÖRFLER AND M. RUMPF
}

\begin{abstract}
We derive a posteriori error estimates for the approximation of linear elliptic problems on domains with piecewise smooth boundary. The numerical solution is assumed to be defined on a Finite Element mesh, whose boundary vertices are located on the boundary of the continuous problem. No assumption is made on a geometrically fitting shape.

A posteriori error estimates are given in the energy norm and the $L^{2}$-norm, and efficiency of the adaptive algorithm is proved in the case of a saturated boundary approximation. Furthermore, a strategy is presented to compute the effect of the non-discretized part of the domain on the error starting from a coarse mesh. This especially implies that parts of the domain, where the measured error is small, stay non-discretized. The presented algorithm includes a stable path following to supply a sufficient polygonal approximation of the boundary, the reliable computation of the a posteriori estimates and a mesh adaptation based on Delaunay techniques. Numerical examples illustrate that errors outside the initial discretization will be detected.
\end{abstract}

\section{INTRODUCTION}

Adaptive Finite Element methods to solve partial differential equations numerically are widely used. Their theoretical foundation is a so called a posteriori error estimate, that is a computable estimate for the error in a suitable norm in terms of the actual numerical solution and data of the problem. Such an estimate allows the computation of a solution with a prescribed error tolerance. The local contributions to this error estimate can be used to judge the local error distribution and hence to mark a set of elements where further refinement of the mesh is necessary.

Usually, it is assumed that the error from the representation of data and the polygonal approximation of the boundary is negligible. But these assumptions could lead to wrong results in practice since they are not fulfilled on coarse grids. In the present paper we will focus on the effect of boundary discretization on the error. So far, only the case of coinciding discrete and continuous boundary has been considered in the a posteriori analysis. From the vast literature on this subject we cite [BW, BR, Ve1, Ve2]. A robust algorithm which takes into account the error due to unavoidable data approximation is described and analyzed in [Dö1].

An a posteriori error analysis for the approximation of linear elliptic problems on domains with piecewise smooth boundary is given. The numerical solution is assumed to be defined on a Finite Element mesh, whose boundary vertices are

Received by the editor March 4, 1996 and, in revised form, January 23, 1997.

1991 Mathematics Subject Classification. Primary 65N15, 65N30, 65N50.

Key words and phrases. Adaptive mesh refinement, a posteriori error estimate, boundary approximation, Poisson's equation.

(C)1998 American Mathematical Society 
located on the boundary of the continuous problem. We derive a posteriori error estimates for the error in the energy and $L^{2}$-norm without assumptions on the quality of the approximation of $\Omega$ by $\Omega_{h}$.

We will observe that those terms in the a posteriori error analysis which are due to the boundary approximation depend on the boundary geometry and on prescribed data. Although the boundary geometry may be quite complicated, a coarse approximation may be sufficient if there is no additional structure in the solution due to prescribed data. On the other hand, even very "smooth" data along the boundary can cause large error terms due to the boundary shape; for instance, at bottlenecks in the domain. So we ask for an adaptive strategy that is capable of noticing that, for a given error tolerance, a quite coarse approximation of $\Omega$ is sufficient or that even large parts of the domain can stay non-discretized.

As a model problem we will consider the Dirichlet problem for Poisson's equation on some bounded domain $\Omega \subset \mathbb{R}^{n}$ with piecewise smooth boundary, prescribed right hand side and boundary values. Motivated by the requirements from realistic applications, we assume that data can be requested at arbitrary points in the domain and on the boundary respectively. No explicit extension of boundary data is required, and the implementation should manage with functions evaluating right hand side and boundary data pointwise.

We then describe an implementation of a robust and efficient algorithm in two space dimensions based on linear Finite Elements.

The algorithm starts with a geometrical approximation of the boundary of $\Omega$. Here we use an adaptive path following strategy that computes a polygonal approximation of $\partial \Omega$, where $\partial \Omega$ is assumed to be implicitly defined by a set of smooth functions.

The error terms that come from the non-discretized part of the domain are computed efficiently. Here, efficient means that the computing cost to estimate errors due to non-discretized parts of the domain is of lower order compared with the cost of an overall regular approximation of the domain. The local estimates include the testing of data at random points which helps in detecting local errors reliably.

In examples we demonstrate that starting from a very coarse approximation of $\Omega$ our method is capable of computing numerical solutions even if essential error terms come from the non-discretized part of the domain and that regions with small error contribution will stay non-discretized.

The organization of the paper is as follows. Sections 2 and 3 are devoted to notations, problem setting and main assumptions. In Section 4 we prove a generalized interpolation result, and Section 5 contains the main theoretical results. Section 6 gives computable upper bounds for some Sobolev embedding constants, which occur in the estimates, in terms of the underlying domain. In Section 7 we describe a robust implementation in two dimensions, including path following, mesh

generation, refinement and local error estimation. Finally, several computational results are given in Section 8.

\section{Notations}

Let $G$ be a bounded domain in $\mathbb{R}^{n}(n=1,2,3)$ with piecewise smooth boundary. By $d_{G}$ we denote the diameter of $G$. For $m \in \mathbb{N}_{0}$ and $p \geq 1$ let $H^{m, p}(G)$ be the well-known Sobolev spaces [Ad], by notational convention $H^{0, p}(G):=L^{p}(G)$. The 
norm of $v \in L^{p}(G)$, respectively $v \in H^{m, p}(G)$, is defined by

$$
\|v\|_{0, p ; G}:=\left(\int_{G}|v|^{p}\right)^{\frac{1}{p}}, \quad\|v\|_{m, p ; G}:=\sum_{s=0}^{m}\left\|\nabla^{s} v\right\|_{0, p ; G} .
$$

In case $p=2$, we will simply write $\|\cdot\|_{m ; G}$.

Fix any domain $\Omega \subset \mathbb{R}^{n}(n=2,3)$ with the above properties. Let $\Omega_{h}$ be a domain with polygonal boundary and $\mathcal{T}_{h}$ be a regular decomposition (discretization) of $\overline{\Omega_{h}}$ into closed $n$-simplices [Ci, Ch. $2, \S 2.1, \S 2.2$ ] such that each vertex on $\partial \Omega_{h}$ is also an element of $\partial \Omega\left(\mathcal{T}_{h}^{\partial}\right.$ is the set of simplices that have nontrivial intersection with the boundary and $\left.\mathcal{T}_{h}^{\circ}:=\mathcal{T}_{h} \backslash \mathcal{T}_{h}^{\partial}\right)$. By $\mathcal{E}_{h}$ we denote the set of faces $\left(\mathcal{E}_{h}^{\partial}\right.$ the faces which are part of $\left.\partial \Omega_{h}, \mathcal{E}_{h}^{\circ}:=\mathcal{E}_{h} \backslash \mathcal{E}_{h}^{\partial}\right)$.

To $\mathcal{T}_{h}$ we assign the shape constant

$$
\sigma_{h}:=\max \left\{\max _{T \in \mathcal{T}_{h}} \frac{d_{T}}{\rho_{T}}, \max _{T, T^{\prime} \in \mathcal{T}_{h}: T \cap T^{\prime} \neq \emptyset} \frac{d_{T}}{d_{T^{\prime}}}\right\},
$$

where $\rho_{T}$ denotes the radius of the largest inner ball of $T$.

Let $V_{h}$ be a Lagrangian finite element space on $\Omega_{h}$ as defined in [Ci, Ch. 2, $\S 2.3]$. Elements of $V_{h}$ are assumed to be piecewise polynomials (with respect to the discretization $\mathcal{T}_{h}$ ) of maximal degree $M \geq 1$ and globally continuous; hence $V_{h} \subset H^{1,2}\left(\Omega_{h}\right) . \quad V_{h}$ is characterized by a set of nodes $p \in \mathcal{N}_{h}$ with $p \in \overline{\Omega_{h}}$. We assume that each element $v$ of $V_{h}$ is uniquely determined by the values on $p \in \mathcal{N}_{h}$. Thus $\psi_{p}(q)=\delta_{p q}$ defines a basis $\left\{\psi_{p}\right\}_{p \in \mathcal{N}_{h}}$ of $V_{h}$, called the nodal basis.

Define $\mathcal{N}_{h}^{\partial}$ to be the set of nodes $p$ contained in $\partial \Omega_{h}$. Let $\stackrel{\circ}{V}_{h}$ be the linear space

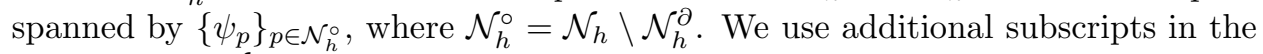
notations for $\mathcal{N}_{h}, \mathcal{E}_{h}$ to describe certain subsets:

$$
\begin{aligned}
\mathcal{N}_{h ; T} & :=\left\{p \in \mathcal{N}_{h} \mid p \in T\right\}, \\
\mathcal{E}_{h ; T} & :=\left\{E \in \mathcal{E}_{h} \mid E \subset \partial T\right\} .
\end{aligned}
$$

Furthermore denote by $\omega_{T}, \omega_{p}, \omega_{E}$, for $T \in \mathcal{T}_{h}, p \in \mathcal{N}_{h}, E \in \mathcal{E}_{h}$, respectively, the following subsets of $\overline{\Omega_{h}}$ :

$$
\begin{aligned}
\omega_{T} & :=\left\{x \in \overline{\Omega_{h}} \mid x \in T^{\prime}, T^{\prime} \in \mathcal{T}_{h}, T \cap T^{\prime} \neq \emptyset\right\}, \\
\omega_{p} & :=\left\{x \in \overline{\Omega_{h}} \mid x \in T^{\prime}, T^{\prime} \in \mathcal{T}_{h}, p \in T^{\prime}\right\}, \\
\omega_{E} & :=\left\{x \in \overline{\Omega_{h}} \mid x \in T^{\prime}, T^{\prime} \in \mathcal{T}_{h}, E \subset \partial T^{\prime}\right\} .
\end{aligned}
$$

In terms of this notation we obtain for the nodal basis $\operatorname{supp} \psi_{p} \subset \omega_{p}$. Since in general $\Omega \neq \Omega_{h}$, we have to provide extensions for functions on $\Omega$ to $\Omega_{h}$ and vice versa. If $v \in \stackrel{\circ}{H}^{1,2}(\Omega)$, we extend it by zero outside $\Omega$ to get a function in $H^{1,2}\left(\Omega_{h}\right)$ (an analogous extension is given if we exchange $\Omega$ and $\Omega_{h}$ ). For notational convenience, we will denote this extended function again by $v$. If $v_{h} \in V_{h}$, we assume that we have a uniquely defined extension $\tilde{v}_{h} \in H^{1,2}\left(\Omega \cup \Omega_{h}\right)$ such that $\tilde{v}_{h}=v_{h}$ on $\Omega_{h}$ and $\tilde{v}_{h}$ is smooth on connected components of $\Omega \backslash \Omega_{h}$. Let us denote the space of these extended functions by $\tilde{V}_{h}$. For the following, we will make the assumption that $\bar{\Omega} \backslash \Omega_{h}$ is decomposed into disjoined closed subsets:

$$
\bar{\Omega} \backslash \Omega_{h}=\bigcup_{E \in \mathcal{E}_{h}^{\partial}} \Omega^{E}, \quad \stackrel{\circ}{ }^{E} \cap \stackrel{\circ}{ }^{E^{\prime}}=\emptyset \quad \text { for } E \neq E^{\prime} .
$$

That is, to each $E \in \mathcal{E}_{h}^{\partial}$ there is a (not necessarily connected) non-discretized closed subset $\Omega^{E}$ of $\bar{\Omega}$ with piecewise smooth boundary (see Figure 1 ). It is assumed that 
$\partial \Omega^{E} \cap \partial \Omega$ and $\partial \Omega^{E} \cap E$ have non-vanishing $n$-1-dimensional measure for each connected component of $\Omega^{E}$. Thus $\tilde{\mathcal{T}}_{h}:=\mathcal{T}_{h} \cup\left\{\Omega^{E} \mid E \in \mathcal{E}_{h}^{\partial}\right\}$ is a decomposition of $\overline{\Omega \cup \Omega_{h}}$. Furthermore, for later usage we define $\tilde{\mathcal{E}}_{h}:=\mathcal{E}_{h} \cup\left\{\left(\partial \Omega^{E} \backslash E\right) \cap \Omega \mid E \in \mathcal{E}_{h}^{\partial}\right\}$, and $\tilde{\omega}_{E}:=\omega_{E} \cup \Omega^{E}$ for $E \in \mathcal{E}_{h}^{\partial}$. In two space dimensions the set $\Omega^{E}$ for $E \in \mathcal{E}_{h}^{\partial}$ can be uniquely defined as the closure of the union of connected components of $\Omega \backslash \Omega_{h}$, that have a non-vanishing intersection (with respect to $n$-1-dimensional measure) with $E$, because of our assumption that boundary vertices of $\mathcal{T}_{h}$ are located on $\partial \Omega$. By that choice there are no extra edges outside $\mathcal{T}_{h}$, and $\tilde{\mathcal{E}}_{h}$ coincides with $\mathcal{E}_{h}$. In three dimensions the edges of boundary faces are generally not contained in $\partial \Omega$. This leads to an ambiguity in the definition of the sets $\Omega^{E}$ (for a hint on a possible constructive definition see Section 7).

\section{THE MODEL PROBLEM}

As a model problem let us consider the Poisson problem with Dirichlet boundary conditions. That is, for given domain $\Omega$ (as above), sufficiently smooth functions $f: \Omega \rightarrow \mathbb{R}, g^{\partial}: \partial \Omega \rightarrow \mathbb{R}$ we seek a function $u: \Omega \rightarrow \mathbb{R}$ (having at least two continuous derivatives) such that $-\Delta u=f$ in $\Omega$ and $u=g^{\partial}$ on $\partial \Omega$. In variational form this problem reads as follows:

Problem (P). For $f \in L^{2}(\Omega), g^{\partial} \in H^{\frac{1}{2}, 2}(\partial \Omega)$ find $u \in H^{1,2}(\Omega)$ such that

$$
\int_{\Omega} \nabla u \cdot \nabla \phi=\int_{\Omega} f \phi \quad \forall \phi \in \stackrel{\circ}{H}^{1,2}(\Omega)
$$

and $u=g^{\partial}$ on $\partial \Omega$.

(For a definition of Sobolev spaces $H^{m, p}$ with positive real $m$ see [Ad].) This problem admits a unique solution by the Riesz-representation theorem. Under appropriate assumptions on $f, g^{\partial}$ and $\Omega$ one can prove that $u$ has two (weak or classical) derivatives $[\mathrm{LM}]$.

The form of the discrete problem is similar: assume that approximations $f_{h}$ of $f$ and $g_{h}^{\partial}$ of $g^{\partial}$ are given such that $g_{h}^{\partial}$ is the trace of some $g_{h} \in V_{h}$ on $\partial \Omega_{h}$.

Problem $\left(\mathbf{P}_{\mathbf{h}}\right)$. For $f_{h} \in L^{2}\left(\Omega_{h}\right), g_{h}^{\partial}$ as above, find $u_{h} \in V_{h}$ such that

$$
\int_{\Omega_{h}} \nabla u_{h} \cdot \nabla \phi_{h}=\int_{\Omega_{h}} f_{h} \phi_{h} \quad \forall \phi_{h} \in \stackrel{\circ}{V}_{h}
$$

and $u_{h}=g_{h}^{\partial}$ on $\partial \Omega_{h}$.

Testing this equality on the nodal basis (see Section 2), we arrive at a sparse positive symmetric system of equations that can be solved efficiently (see Section 7).

If problem (P) admits an $H^{2,2}$-regular solution and suitable approximations $f_{h}$ and $g_{h}^{\partial}$ are used, the following a priori estimate holds in case $M=1$ :

$$
\left\|\nabla\left(u-\tilde{u}_{h}\right)\right\|_{0 ; \Omega} \leq C h_{\max }\|u\|_{2 ; \Omega},
$$

where $h_{\max }:=\max _{T \in \mathcal{T}_{h}} d_{T}$ and $C$ depends on $\Omega$ and $\sigma_{h}$ only [BK].

Lemma 1 (Sobolev inequalities). Let $G$ as in Section 2 and $S \subseteq \partial G$ be a set with non-vanishing $n$-1-dimensional measure. 
i. There is a constant $C_{P}(G, S)>0$ such that for all $v \in H^{1,2}(G)$ with $v \equiv 0$ on $S$

$$
\|v\|_{0 ; G} \leq C_{P}(G, S)\|\nabla v\|_{0 ; G} .
$$

If $S=\partial G$, we let $C_{P}(G):=C_{P}(G, \partial G) . C_{P}(G)$ is called Poincaré's constant. ii. There is a constant $C_{\partial}(G)$ such that for all $v \in H^{1,2}(G)$

$$
\|v\|_{0 ; \partial G} \leq C_{\partial}(G)\left(\|\nabla v\|_{0 ; G}+d_{G}^{-1}\|v\|_{0 ; G}\right) .
$$

iii. With $S$ as above, there is a number $C_{\partial}^{\prime}(G, S)$ such that for all $v \in H^{1,2}(G)$ with $v \equiv 0$ on $S$

$$
\|v\|_{0 ; \partial G} \leq C_{\partial}^{\prime}(G, S)\|\nabla v\|_{0 ; G} .
$$

For the proof we refer to $[\mathrm{Ad}, 5.22,6.26]$ and $[\mathrm{Al}, \mathrm{A} 5.7]$.

\section{INTERPOLATION ESTIMATES}

In order to prove error estimates, it is necessary to refer to a (continuous) projection operator from $\stackrel{\circ}{H}^{1,2}(\Omega)$ into the test space $\stackrel{\circ}{V}_{h}$. We use a construction given in $[\mathrm{Cl}]$ to define projections

$$
I_{h}: \stackrel{\circ}{H}^{1,2}(\Omega) \longrightarrow V_{h}, \quad I_{h}^{0}: \stackrel{\circ}{H}^{1,2}(\Omega) \longrightarrow \stackrel{\circ}{V}_{h}
$$

as follows: for $p \in \mathcal{N}_{h}$ let $\gamma_{p}$ be the best $L^{2}$-approximation of $v$ in $\mathbb{P}_{M}$ (the space of polynomials up to degree $M$ ) on $\operatorname{supp} \psi_{p}$. Then define:

$$
\begin{aligned}
& \left(I_{h} v\right)(p):=\gamma_{p}(p) \text { for all } p \in \mathcal{N}_{h}, \\
& \left(I_{h}^{0} v\right)(p):= \begin{cases}\gamma_{p}(p) & \text { for } p \in \mathcal{N}_{h}^{\circ}, \\
0 & \text { for } p \in \mathcal{N}_{h}^{\partial} .\end{cases}
\end{aligned}
$$

(Recall that $v$ is extended by zero outside $\Omega$.) In terms of the set of nodal basis functions $\left\{\psi_{p}\right\}_{p \in \mathcal{N}_{h}}$ (see Section 2), we have the following representations:

$$
I_{h} v=\sum_{p \in \mathcal{N}_{h}} \gamma_{p}(p) \psi_{p}, \quad I_{h}^{0} v=\sum_{p \in \mathcal{N}_{h}^{\circ}} \gamma_{p}(p) \psi_{p} .
$$

Since $\Omega_{h} \neq \Omega$, we cannot directly apply the estimates for $v-I_{h}^{0} v$ proved in [Cl]. Before stating the generalizations for the present situation, some additional notation is needed.

Definition 1. $i$. For any $\mathcal{T} \subseteq \tilde{\mathcal{T}}_{h}$ and $q \in[1, \infty]$ we define

$$
L^{q}(\mathcal{T}):=\left\{\left(w_{T}\right)_{T \in \mathcal{T}} \mid w_{T} \in L^{q}(T) \quad \forall T \in \mathcal{T}\right\} .
$$

For $v, w \in L^{2}(\mathcal{T})$ we define a scalar product, respectively norm, by

$$
(v, w)_{\mathcal{T}}:=\sum_{T \in \mathcal{T}} \int_{T} v_{T} w_{T}, \quad\|v\|_{\mathcal{T}}:=\left(\sum_{T \in \mathcal{T}}\left\|v_{T}\right\|_{0 ; T}^{2}\right)^{\frac{1}{2}} .
$$

If $\Omega_{\mathcal{T}}$ is the domain that is composed of all $T \in \mathcal{T}$, we can identify $w \in L^{2}\left(\Omega_{\mathcal{T}}\right)$ with $\left(\left.w\right|_{T}\right)_{T \in \mathcal{T}} \in L^{2}(\mathcal{T})$ and therefore write $\int_{\Omega_{\mathcal{T}}} v w=(v, w)_{\mathcal{T}}$ for $v, w \in$ $L^{2}\left(\Omega_{\mathcal{T}}\right)$. In the same way let us define for any set $\mathcal{E} \subseteq \tilde{\mathcal{E}}_{h}$

$$
L^{q}(\mathcal{E}):=\left\{\left(w_{E}\right)_{E \in \mathcal{E}} \mid w_{E} \in L^{q}(E) \quad \forall E \in \mathcal{E}\right\}
$$


with corresponding scalar product, respectively norm $(q=2)$ :

$$
(v, w)_{\mathcal{E}}:=\sum_{E \in \mathcal{E}} \int_{E} v_{E} w_{E}, \quad\|v\|_{\mathcal{E}}:=\left(\sum_{E \in \mathcal{E}}\left\|v_{E}\right\|_{0 ; E}^{2}\right)^{\frac{1}{2}} .
$$

ii. For $v_{h} \in \tilde{V}_{h}$ let $\left[\partial_{h} v_{h}\right]_{E}$ be the jump of the normal derivative of $v_{h}$ on some $E \in \tilde{\mathcal{E}}_{h}$, that is, if $n$ is a (fixed) unit normal vector on $E$, we define

$$
\left[\partial_{n} v_{h}\right]_{E}(x):= \begin{cases}\lim _{s \rightarrow+0} n \cdot \nabla\left(v_{h}(x+s n)-v_{h}(x-s n)\right), & \text { for } x \in E \cap \Omega, \\ 0 & \text { for } x \in E, x \notin \Omega .\end{cases}
$$

Since $v_{h}$ is piecewise smooth, we have that $\left[\partial_{n} v_{h}\right]:=\left(\left[\partial_{n} v_{h}\right]_{E}\right)_{E \in \tilde{\mathcal{E}}_{h}} \in L^{2}\left(\tilde{\mathcal{E}}_{h}\right)$ and $\left(\left[\partial_{n} v_{h}\right], z\right)_{\tilde{\mathcal{E}}_{h}}$ is defined for arbitrary $z \in H^{1,2}\left(\Omega \cup \Omega_{h}\right)$ since $\left.z\right|_{E} \in L^{2}(E)$ for any $E \in \tilde{\mathcal{E}}_{h}$ (see Lemma 1 ).

iii. We define a mesh-size function $\tilde{h} \in L^{\infty}\left(\tilde{\mathcal{T}}_{h}\right)$ by

$$
\tilde{h}_{T}:= \begin{cases}d_{T} & \text { if } T \in \mathcal{T}_{h}^{\circ}, \\ d_{T}\left(1+\sum_{p \in \mathcal{N}_{h ; T}^{\partial}} \min _{E \in \mathcal{E}_{h ; p}^{\partial}} C_{\partial}^{E} d_{E}^{-\frac{1}{2}}\right) & \text { if } T \in \mathcal{T}_{h}^{\partial}, \\ C_{P}^{E} & \text { if } T=\Omega^{E} \text { for some } E \in \mathcal{E}_{h}^{\partial},\end{cases}
$$

where (for notation see Lemma 1)

$$
\begin{aligned}
& \mathcal{E}_{h ; p}^{\partial}:=\left\{E \in \mathcal{E}_{h}^{\partial} \mid p \in E\right\}, \\
& C_{P}^{E}:=C_{P}\left(\Omega^{E}, \partial \Omega^{E} \cap \partial \Omega\right), C_{\partial}^{E}:=C_{\partial}^{\prime}\left(\Omega^{E}, \partial \Omega^{E} \cap \partial \Omega\right) .
\end{aligned}
$$

Note that the existence of finite constants $C_{P}^{E}, C_{\partial}^{E}$ is guaranteed by our assumptions on $\Omega^{E}$ (see Section 2).

$i v$. For $v_{h} \in \tilde{V}_{h}$ define

$$
\begin{aligned}
\tilde{D}_{1}^{2}\left(v_{h}\right) & :=\left(\sum_{T \in \mathcal{T}_{h}} d_{T}^{-1} \tilde{h}_{T}^{2}\left\|\left[\partial_{n} v_{h}\right]\right\|_{\mathcal{E}_{h ; T}^{\circ}}^{2}\right)^{\frac{1}{2}}, \\
\tilde{D}_{1 ; \partial}^{2}\left(v_{h}\right) & :=\left(\sum_{E \in \mathcal{E}_{h}^{\partial}} C_{\partial}^{E^{2}}\left\|\left[\partial_{n} v_{h}\right]\right\|_{0 ; \partial \Omega^{E} \cap \Omega}^{2}\right)^{\frac{1}{2}} .
\end{aligned}
$$

Lemma 2 (Interpolation estimates). There are constants $c_{1}, c_{2}$, depending only on the shape constant $\sigma_{h}, n$, and on $M$, such that for all $w \in L^{2}\left(\tilde{\mathcal{T}}_{h}\right), z_{h} \in \tilde{V}_{h}$, and $v \in \stackrel{\circ}{H}^{1,2}(\Omega)$,

$$
\begin{aligned}
\left|\left(w, v-I_{h}^{0} v\right)_{\tilde{\mathcal{T}}_{h}}\right| & \leq\left(c_{1}\|\tilde{h} w\|_{\mathcal{T}_{h}}+\|\tilde{h} w\|_{\tilde{\mathcal{T}}_{h} \backslash \mathcal{T}_{h}}\right)\|\nabla v\|_{0 ; \Omega}, \\
\left|\left(\left[\partial_{n} z_{h}\right], v-I_{h}^{0} v\right)_{\tilde{\mathcal{E}}_{h}}\right| & \leq\left(c_{2} \tilde{D}_{1}^{2}\left(z_{h}\right)+\tilde{D}_{1, \partial}^{2}\left(z_{h}\right)\right)\|\nabla v\|_{0 ; \Omega} .
\end{aligned}
$$

Proof. We start with the following decomposition (recall that $v \equiv 0$ outside $\Omega$ and $I_{h}^{0} v \equiv 0$ outside $\left.\Omega_{h}\right)$ :

$$
\begin{aligned}
\left(w, v-I_{h}^{0} v\right)_{\tilde{\mathcal{T}}_{h}} & =\left(w, v-I_{h}^{0} v\right)_{\mathcal{T}_{h}}+(w, v)_{\tilde{\mathcal{T}}_{h} \backslash \mathcal{T}_{h}} \\
& =\left(w, v-I_{h} v\right)_{\mathcal{T}_{h}}+\left(w, I_{h} v-I_{h}^{0} v\right)_{\mathcal{T}_{h}}+(w, v)_{\tilde{\mathcal{T}}_{h} \backslash \mathcal{T}_{h}} .
\end{aligned}
$$


Using the result in $[\mathrm{Cl}]$, the first term on the right hand side can be estimated as follows:

$$
\left|\left(w, v-I_{h} v\right)_{\mathcal{T}_{h}}\right| \leq c\|\tilde{h} w\|_{\mathcal{T}_{h}}|| \nabla v \|_{0 ; \Omega_{h}}
$$

(note that $\tilde{h} \geq d_{T}$ for $T \in \mathcal{T}_{h}$ ). To estimate the third term, we recall that $\tilde{\mathcal{T}}_{h} \backslash \mathcal{T}_{h}$ consists of sets $\Omega^{E}$ for $E \in \mathcal{E}_{h}^{\partial}$. Since $v$ vanishes on $\partial \Omega^{E} \cap \partial \Omega$, we have

$$
\|v\|_{0 ; \Omega^{E}} \leq C_{P}^{E}\|\nabla v\|_{0 ; \Omega^{E}}
$$

and this gives

$$
\left|(w, v)_{\tilde{\mathcal{T}}_{h} \backslash \mathcal{T}_{h}}\right| \leq \sum_{E \in \mathcal{E}_{h}^{\partial}}\left|\int_{\Omega^{E}} w v\right| \leq \sum_{E \in \mathcal{E}_{h}^{\partial}} C_{P}^{E}\|w\|_{0 ; \Omega^{E}}\|\nabla v\|_{0 ; \Omega^{E}} .
$$

Since $I_{h} v-I_{h}^{0} v$ vanishes on elements in $\mathcal{T}_{h}^{\circ}$, we have for the second term

$$
\left|\left(w, I_{h} v-I_{h}^{0} v\right)_{\mathcal{T}_{h}}\right| \leq \sum_{T \in \mathcal{T}_{h}^{\partial}}\|w\|_{0 ; T}\left\|I_{h} v-I_{h}^{0} v\right\|_{0 ; T} .
$$

By definition of the interpolation operators we have

$$
\left\|I_{h} v-I_{h}^{0} v\right\|_{0 ; T} \leq \sum_{p \in \mathcal{N}_{h ; T}^{\partial}}\left\|\gamma_{p}(p) \psi_{p}\right\|_{0 ; T} \leq c d_{T}^{\frac{n}{2}} \sum_{p \in \mathcal{N}_{h ; T}^{\partial}} \min _{E \in \mathcal{E}_{h ; p}^{\partial}}\left\|\gamma_{p}\right\|_{0, \infty ; E} .
$$

Since $\gamma_{p}$ is a polynomial, we can estimate its $L^{\infty}$-norm by its $L^{2}$-norm on the rescaled face $E / d_{E}$ (independently of $\gamma_{p}$ ). Note further that $\gamma_{p}$ also obeys the inequalities $\left\|\nabla^{s} \gamma_{p}\right\|_{0 ; T} \leq c\left\|\nabla^{s} v\right\|_{0 ; \omega_{T}}$ for $s \in\{0,1\}$ and $T \in \mathcal{T}_{h}$. For each $E \in \mathcal{E}_{h}^{\partial}$ let $T(E)$ be the unique element in $\mathcal{T}_{h}^{\partial}$ with $E \subset \partial T(E)$. Then

$$
\begin{aligned}
\left\|\gamma_{p}\right\|_{0, \infty ; E} \leq c d_{E}^{-\frac{n-1}{2}}\left\|\gamma_{p}\right\|_{0 ; E} \leq c d_{E}^{-\frac{n-1}{2}}\left(\|v\|_{0 ; E}+\left\|v-\gamma_{p}\right\|_{0 ; E}\right) \\
\leq c d_{E}^{-\frac{n-1}{2}}\left(C_{\partial}^{E}\|\nabla v\|_{0 ; \Omega^{E}}\right. \\
\left.\quad+c d_{T(E)}^{\frac{1}{2}}\left(\left\|\nabla\left(v-\gamma_{p}\right)\right\|_{0 ; T(E)}+d_{T(E)}^{-1}\left\|v-\gamma_{p}\right\|_{0 ; T(E)}\right)\right) \\
\leq c d_{E}^{-\frac{n-1}{2}}\left(C_{\partial}^{E}\|\nabla v\|_{0 ; \Omega^{E}}+d_{T(E)}^{\frac{1}{2}}\|\nabla v\|_{0 ; \omega_{T(E)}}\right) .
\end{aligned}
$$

Here we use again the results of $[\mathrm{Cl}]$ and Lemma 1 on the rescaled triangle $T(E) / d_{T}(E)$. Thus

$$
\begin{aligned}
& \left|\left(w, v-I_{h}^{0} v\right)_{\tilde{\mathcal{T}}_{h}}\right| \\
& \leq c|| \tilde{h} w\left\|_{\mathcal{T}_{h}}\right\| \nabla v\left\|_{0 ; \Omega}+\sum_{E \in \mathcal{E}_{h}^{\partial}} C_{P}^{E}\right\| w\left\|_{0 ; \Omega^{E}}\right\| \nabla v \|_{0 ; \Omega^{E}} \\
& \quad+c \sum_{T \in \mathcal{T}_{h}^{\partial}} d_{T}\|w\|_{0 ; T}\left(\|\nabla v\|_{0 ; \omega_{T}}+\sum_{p \in \mathcal{N}_{h ; T}^{\partial}} \min _{E \in \mathcal{E}_{h ; p}^{\partial}} C_{\partial}^{E} d_{E}^{-\frac{1}{2}}\|\nabla v\|_{0 ; \Omega^{E}}\right) \\
& \leq c\|\tilde{h} w\|_{\mathcal{T}_{h}}\|\nabla v\|_{0 ; \Omega}+\left(\sum_{E \in \mathcal{E}_{h}^{\partial}} C_{P}^{E^{2}}\|w\|_{0 ; \Omega^{E}}^{2}\right)^{\frac{1}{2}}\|\nabla v\|_{0 ; \Omega \backslash \Omega_{h}},
\end{aligned}
$$




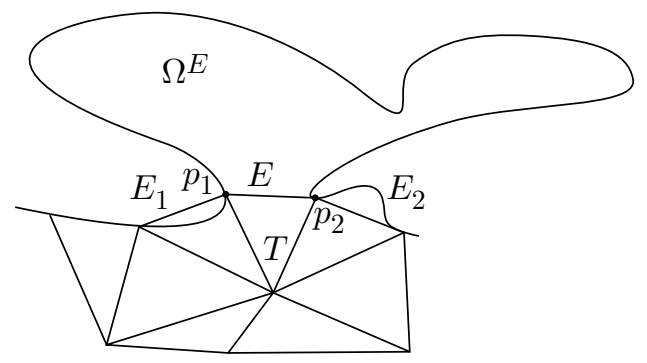

FiguRE 1. In the estimate of the interpolation error $I_{h}^{0} v-I_{h} v$ we are allowed to choose a face $E$ with $p \in \mathcal{N}_{h ; E}$ such that $C_{\partial}^{E}$ gets small. In the above example we see that for node $p_{2}$ in $\mathcal{N}_{h ; T}$ the constant $C_{\partial}^{E_{2}}$ will be much smaller than $C_{\partial}^{E}$ and for $p_{1}$ the constant $C_{\partial}^{E_{1}}$ already vanishes.

and this leads immediately to the first assertion of the lemma. To prove the second inequality, we proceed as in the beginning of the proof:

$$
\begin{aligned}
& \left|\left(\left[\partial_{n} u_{h}\right], v-I_{h}^{0} v\right)_{\tilde{\mathcal{E}}_{h}}\right| \\
& \leq \sum_{E \in \mathcal{E}_{h}^{\circ}}\left\|\left[\partial_{n} u_{h}\right]\right\|_{0 ; E}\left\|v-I_{h}^{0} v\right\|_{0 ; E}+\sum_{E \in \mathcal{E}_{h}^{\partial}}\left\|\left[\partial_{n} u_{h}\right]\right\|_{0 ; \partial \Omega^{E} \cap \Omega}\|v\|_{0 ; \partial \Omega^{E} \cap \Omega} \\
& \leq \sum_{T \in \mathcal{T}_{h}}\left\|\left[\partial_{n} u_{h}\right]\right\|_{\mathcal{E}_{h ; T}^{\circ}}\left\|v-I_{h} v\right\|_{0 ; \partial T}+\sum_{T \in \mathcal{T}_{h}^{\partial}}\left\|\left[\partial_{n} u_{h}\right]\right\|_{\mathcal{E}_{h ; T}^{\circ}}\left\|I_{h} v-I_{h}^{0} v\right\|_{0 ; \partial T} \\
& \quad+\sum_{E \in \mathcal{E}_{h}^{\partial}}\left\|\left[\partial_{n} u_{h}\right]\right\|_{0 ; \partial \Omega^{E} \cap \Omega}\|v\|_{0 ; \partial \Omega^{E} \cap \Omega} .
\end{aligned}
$$

For the third term we immediately get

$$
\sum_{E \in \mathcal{E}_{h}^{\partial}}\left\|\left[\partial_{n} u_{h}\right]\right\|\left\|_{0 ; \partial \Omega^{E} \cap \Omega}\right\| v\left\|_{0 ; \partial \Omega^{E} \cap \Omega} \leq\left(\sum_{E \in \mathcal{E}_{h}^{\partial}} C_{\partial}^{E^{2}}\left\|\left[\partial_{n} u_{h}\right]\right\| \|_{0 ; \partial \Omega^{E} \cap \Omega}^{2}\right)^{\frac{1}{2}}\right\| \nabla v \|_{0 ; \Omega \backslash \Omega_{h}} .
$$

Using a previous result, we get for the second term

$$
\begin{aligned}
& \sum_{T \in \mathcal{T}_{h}^{\partial}}\left\|\left[\partial_{n} u_{h}\right]\right\|_{\mathcal{E}_{h ; T}^{\circ}}\left\|I_{h} v-I_{h}^{0} v\right\|_{0 ; \partial T} \leq c \sum_{T \in \mathcal{T}_{h}^{\partial}} d_{T}^{-\frac{1}{2}}\left\|\left[\partial_{n} u_{h}\right]\right\|\left\|_{\mathcal{E}_{h ; T}^{\circ}}\right\| I_{h} v-I_{h}^{0} v \|_{0 ; T} \\
& \quad \leq c \sum_{T \in \mathcal{T}_{h}^{\partial}} d_{T}^{\frac{1}{2}}\left\|\left[\partial_{n} u_{h}\right]\right\|_{\mathcal{E}_{h ; T}^{\circ}}\left(\|\nabla v\|_{0 ; \omega_{T}}+\sum_{p \in \mathcal{N}_{h ; T}^{\partial}} \min _{E \in \mathcal{E}_{h ; p}^{\partial}} C_{\partial}^{E} d_{E}^{-\frac{1}{2}}\|\nabla v\|_{0 ; \Omega^{E}}\right),
\end{aligned}
$$

while for the first term we again apply an estimate given in $[\mathrm{Cl}]$ and obtain

$$
\sum_{T \in \mathcal{T}_{h}}\left\|\left[\partial_{n} u_{h}\right]\right\|_{\mathcal{E}_{h ; T}^{\circ}}\left\|v-I_{h} v\right\|_{0 ; \partial T} \leq c \sum_{T \in \mathcal{T}_{h}} d_{T}^{\frac{1}{2}}\left\|\left[\partial_{n} u_{h}\right]\right\|_{\mathcal{E}_{h ; T}^{\circ}}\|\nabla v\|_{0 ; \omega_{T}} .
$$

This proves the second assertion.

Remark 1. i. If $\Omega=\Omega_{h}$, we have $C_{\partial}^{E}=C_{P}^{E}=0$ and get the results of [Cl] for empty sets $\Omega^{E}$.

ii. The numbers $C_{\partial}^{E}, C_{P}^{E}$ both depend on the size of $\Omega^{E}$. Explicit bounds for the case $n=2$ are given in Section 6 . 
iii. Figure 1 gives an example of how the estimates benefit from an appropriate choice of edges in the evaluation of $\tilde{h}$.

$i v$. Our estimates are based on the interpolation operators introduced in $[\mathrm{Cl}]$. A different interpolation using $L^{2}$-projections on faces instead of elements is given in [SZ]. With respect to those one could obtain similar results.

\section{A posteriori ERror ESTIMATES}

Definition 2. The continuous residual $r\left(v_{h}\right) \in L^{2}\left(\tilde{\mathcal{T}}_{h}\right)$ of $v_{h} \in \tilde{V}_{h}$ is defined by

$$
r\left(v_{h}\right)_{T}:= \begin{cases}\left.\Delta v_{h}\right|_{T}+\left.f_{h}\right|_{T} & \text { for } T \in \mathcal{T}_{h}, \\ \left.\Delta \tilde{v}_{h}\right|_{T}+f & \text { for } T \in \tilde{\mathcal{T}}_{h} \backslash \mathcal{T}_{h} .\end{cases}
$$

$\left.\Delta v_{h}\right|_{T}$ means that we take the Laplacian of the polynomial $\left.v_{h}\right|_{T}$, considered as a function defined on $\mathbb{R}^{n}$, evaluated on $T$.

The following theorem generalizes a well-known a posteriori estimate for the case $\Omega=\Omega_{h}$ (see e.g. [Ve1]) to the case $\Omega \neq \Omega_{h}$ under the assumptions made in Section 2 . Note that there is no condition requiring that $\partial \Omega$ should be sufficiently close to $\partial \Omega_{h}$ or that $\partial \Omega$ is locally a graph over $\partial \Omega_{h}$.

Theorem 1 ( $H^{1}$-a posteriori error estimate). Let $u$ and $u_{h}$ be the respective solutions of problems $(\mathrm{P}),\left(\mathrm{P}_{\mathrm{h}}\right)$. Let $\tilde{u}_{h}$ be an extension of $u_{h}$ as described in Section 2, $\tilde{h}$ the grid function defined in Definition 1.iii, $r\left(\tilde{u}_{h}\right)$ the continuous residual (Definition 2), and $\tilde{g}, \tilde{g}_{h} \in H^{1,2}\left(\Omega \cup \Omega_{h}\right)$ with $\tilde{g}=u$ and $\tilde{g}_{h}=\tilde{u}_{h}$ on $\partial \Omega$. Then there are constants $c_{1}, c_{2}$ depending on $\sigma_{h}, n$, and $M$, such that for $e_{h}:=u-\tilde{u}_{h}$

$$
\begin{gathered}
\left\|\nabla e_{h}\right\|_{0 ; \Omega} \leq c_{1}\left\|\tilde{h} r\left(u_{h}\right)\right\|_{\mathcal{T}_{h}}+c_{2} \tilde{D}_{1}^{2}\left(u_{h}\right)+\left\|\tilde{h} r\left(\tilde{u}_{h}\right)\right\|_{\tilde{\mathcal{T}}_{h} \backslash \mathcal{T}_{h}}+\tilde{D}_{1, \partial}^{2}\left(\tilde{u}_{h}\right) \\
+2\left\|\nabla\left(\tilde{g}-\tilde{g}_{h}\right)\right\|_{0 ; \Omega \cup \Omega_{h}}+C_{P}(\Omega)\left\|f-f_{h}\right\|_{0 ; \Omega \cap \Omega_{h}} .
\end{gathered}
$$

Proof. Let $v \in \stackrel{\circ}{H}^{1,2}(\Omega)$ and $v_{h} \in \stackrel{\circ}{V}_{h}$. Then the error is represented by

$$
\begin{aligned}
\int_{\Omega} \nabla e_{h} \cdot \nabla v & =\int_{\Omega} f v-\int_{\Omega} \nabla \tilde{u}_{h} \cdot \nabla v \\
& =\int_{\Omega} f v-\int_{\Omega_{h}} \nabla u_{h} \cdot \nabla\left(v-v_{h}\right)-\int_{\Omega \backslash \Omega_{h}} \nabla \tilde{u}_{h} \cdot \nabla v-\int_{\Omega_{h}} f_{h} v_{h} \\
& =\left(\Delta \tilde{u}_{h}, v-v_{h}\right)_{\tilde{\mathcal{T}}_{h}}+\left(\left[\partial_{h} \tilde{u}_{h}\right], v-v_{h}\right)_{\tilde{\mathcal{E}}_{h}}+\int_{\Omega} f v-\int_{\Omega_{h}} f_{h} v_{h} \\
& =\left(r\left(\tilde{u}_{h}\right), v-v_{h}\right)_{\tilde{\mathcal{T}}_{h}}+\left(\left[\partial_{h} \tilde{u}_{h}\right], v-v_{h}\right)_{\tilde{\mathcal{E}}_{h}}+\int_{\Omega \cap \Omega_{h}}\left(f-f_{h}\right) v .
\end{aligned}
$$

Taking especially $v_{h}=I_{h}^{0} v$ and using Lemmata 1,2 , and Definition 2 we obtain

$$
\begin{aligned}
\left|\int_{\Omega \cap \Omega_{h}}\left(f-f_{h}\right) v\right| & \leq C_{P}(\Omega)\left\|f-f_{h}\right\|_{0 ; \Omega \cap \Omega_{h}}\|\nabla v\|_{0 ; \Omega}, \\
\left|\left(r\left(\tilde{u}_{h}\right), v-v_{h}\right)_{\tilde{\mathcal{T}}_{h}}\right| & \leq\left(c_{1}\left\|\tilde{h} r\left(u_{h}\right)\right\|_{\mathcal{T}_{h}}+\left\|\tilde{h} r\left(\tilde{u}_{h}\right)\right\| \|_{\tilde{\mathcal{T}}_{h} \backslash \mathcal{T}_{h}}\right)\|\nabla v\|_{0 ; \Omega}, \\
\left|\left(\left[\partial_{h} \tilde{u}_{h}\right], v-v_{h}\right)_{\tilde{\mathcal{E}}_{h}}\right| & \leq\left(c_{2} \tilde{D}_{1}^{2}\left(u_{h}\right)+\tilde{D}_{1, \partial}^{2}\left(\tilde{u}_{h}\right)\right)\|\nabla v\|_{0 ; \Omega} .
\end{aligned}
$$


Since $e_{h}-\left(\tilde{g}-\tilde{g}_{h}\right) \in \stackrel{\circ}{H}^{1,2}(\Omega)$, we arrive at

$$
\begin{gathered}
\left\|\nabla\left(e_{h}-\left(\tilde{g}-\tilde{g}_{h}\right)\right)\right\|_{0 ; \Omega} \\
\leq c_{1}\left\|\tilde{h} r\left(u_{h}\right)\right\|_{\mathcal{T}_{h}}+\left\|\tilde{h} r\left(\tilde{u}_{h}\right)\right\|_{\tilde{\mathcal{T}}_{\backslash} \backslash \mathcal{T}_{h}}+c_{2} \tilde{D}_{1}^{2}\left(u_{h}\right)+\tilde{D}_{1, \partial}^{2}\left(\tilde{u}_{h}\right) \\
\quad+\left\|\nabla\left(\tilde{g}-\tilde{g}_{h}\right)\right\|_{0 ; \Omega \cup \Omega_{h}}+C_{P}(\Omega)\left\|f-f_{h}\right\|_{0 ; \Omega \cap \Omega_{h}},
\end{gathered}
$$

from which our assertion immediately follows.

Definition 3 (Saturated state). In the following, we will, for technical simplicity, assume that for each $T \in \mathcal{T}_{h}^{\partial}$ there is at most one face $E \subset \mathcal{E}_{h}^{\partial}$. This is no loss of generality since we may otherwise refine such a simplex by bisection. We say that the approximation $\Omega_{h}$ of $\Omega$ reached the saturated state if for each $T \in \mathcal{T}_{h}^{\partial}$ (and $E$ as above)

$i$. with $T \not \subset \Omega$ there is a $n$-simplex $T^{\prime} \subset T$, constructed from $T$ by a parallel displacement of the face $E$, such that $T^{\prime} \subset \Omega$ and $d_{T^{\prime}} \geq \frac{1}{2} d_{T}$,

ii. there is a $n$-simplex $T^{\prime \prime}, T \subset T^{\prime \prime}$, constructed from $T$ by a parallel displacement of the face $E$, such that $\Omega^{E} \subset T^{\prime \prime}$ and $d_{T^{\prime \prime}} \leq 2 d_{T}$,

iii. $\quad \tilde{h}_{T} \leq 2 d_{T}$ for all $T \in \tilde{\mathcal{T}}_{h}$.

Theorem 2 (Efficiency of the estimated $H^{1}$-error in the saturated state). $i$. Let $\left.u_{h}\right|_{T},\left.f_{h}\right|_{T} \in \mathbb{P}_{M}$ for all $T \in \mathcal{T}_{h}$ and assume that conditions $i$. and iii. of Definition 3 are satisfied. Then

$$
c_{1} \tilde{D}_{1}^{2}\left(u_{h}\right)+c_{2}\left\|\tilde{h} r\left(u_{h}\right)\right\|_{\mathcal{T}_{h}} \leq c\left(\left\|\nabla e_{h}\right\|_{0 ; \Omega}+\left\|\tilde{h}\left(f-f_{h}\right)\right\|_{0 ; \Omega \cap \Omega_{h}}\right),
$$

with a constant $c$ depending on $\sigma_{h}, n$ and $M$ only.

ii. Let $n=2$ and conditions i.-iii. of Definition 3 be satisfied. For $T \in \mathcal{T}_{h}^{\partial}$ define $\tilde{u}_{h}$ respectively $f_{h}$ on $T^{\prime \prime}$ to be the extensions of the corresponding polynomials on $T$. Thus $\tilde{D}_{1, \partial}^{2}\left(\tilde{u}_{h}\right)=0$ and

$$
c_{1} \tilde{D}_{1}^{2}\left(u_{h}\right)+c_{2}\left\|\tilde{h} r\left(u_{h}\right)\right\|_{\mathcal{T}_{h}}+\left\|\tilde{h} r\left(\tilde{u}_{h}\right)\right\|_{\tilde{\mathcal{T}}_{h} \backslash \mathcal{T}_{h}} \leq c\left(\left\|\nabla e_{h}\right\|_{0 ; \Omega}+\left\|\tilde{h}\left(f-f_{h}\right)\right\|_{0 ; \Omega}\right),
$$

with a constant $c$ depending on $\sigma_{h}$ and $M$ only.

Proof. $i$. For any $E \in \mathcal{E}_{h}^{\circ}$ and $\phi \in \stackrel{\circ}{H}^{1,2}\left(\omega_{E} \cap \Omega\right)$ the following holds:

$$
\begin{aligned}
\int_{E}\left[\partial_{n} u_{h}\right] \phi-\sum_{T: T \subseteq \omega_{E}} \int_{T}\left(\Delta u_{h}+f_{h}\right) \phi & =\int_{\omega_{E}} \nabla u_{h} \cdot \nabla \phi-\int_{\omega_{E}} f_{h} \phi \\
& =\int_{\omega_{E}} \nabla\left(u_{h}-u\right) \cdot \nabla \phi+\int_{\omega_{E}}\left(f-f_{h}\right) \phi .
\end{aligned}
$$

If $T \subset \Omega$, we can follow the arguments in [Ve1] to get

$$
d_{T}^{\frac{1}{2}}\left\|\left[\partial_{n} u_{h}\right]\right\|_{\mathcal{E}_{h ; T}^{\circ}}+d_{T}\left\|\Delta u_{h}+f_{h}\right\|_{0 ; T} \leq c\left(\left\|\nabla e_{h}\right\|_{0 ; \omega_{T}}+d_{T}\left\|f-f_{h}\right\|_{0 ; \omega_{T}}\right),
$$

where the constant $c$ depends on $\sigma_{h}$ and $M$.

In case $T \not \subset \Omega$ we can use the same arguments as before on $T^{\prime}$ instead of $T$ to get

$$
\begin{aligned}
& d_{T^{\prime}}^{\frac{1}{2}}\left(\sum_{E \in \mathcal{E}_{h ; T}^{\circ}}\left\|\left[\partial_{h} u_{h}\right]\right\|_{0 ; E \cap T^{\prime}}^{2}\right)^{\frac{1}{2}}+d_{T^{\prime}}\left\|\Delta u_{h}+f_{h}\right\|_{0 ; T^{\prime}} \\
& \quad \leq c\left(\left\|\nabla e_{h}\right\|_{0 ; \omega_{T} \cap \Omega}+d_{T}\left\|f-f_{h}\right\|_{0 ; \omega_{T} \cap \Omega}\right) .
\end{aligned}
$$


But by assumptions i. and iii. of Definition 3 and since $\left[\partial_{n} u_{h}\right]$ and $\Delta u_{h}+f_{h}$ are polynomials, we can estimate these terms on $T^{\prime}$ from below by the corresponding terms on $T$.

ii. For $T \in \mathcal{T}_{h}$ and $E \in \mathcal{E}_{h ; T}$ we can proceed as before, taking into account

$$
\left\|\tilde{h} r\left(\tilde{u}_{h}\right)\right\|_{0 ; \Omega^{E}} \leq\left\|\tilde{h}\left(\Delta \tilde{u}_{h}+f_{h}\right)\right\|_{0 ; \Omega^{E}}+\left\|\tilde{h}\left(f-f_{h}\right)\right\|_{0 ; \Omega^{E}},
$$

and estimate

$$
\left\|\tilde{h}\left(\Delta \tilde{u}_{h}+f_{h}\right)\right\|_{0 ; \Omega^{E}} \leq c d_{T^{\prime \prime}}\left\|\Delta \tilde{u}_{h}+f_{h}\right\|_{0 ; T^{\prime \prime}} \leq c d_{T^{\prime}}\left\|\Delta u_{h}+f_{h}\right\|_{0 ; T^{\prime}} .
$$

Remark 2. $i$. In the special case where $\left.f_{h}\right|_{T}$ is the local $L^{2}$-projection of $f$ on $T \cap \Omega$ we can (in Theorems 1 and 2) estimate

$$
\left|\int_{\Omega \cap \Omega_{h}}\left(f-f_{h}\right) v\right| \leq C\left(\sum_{T \in \mathcal{T}_{h}} d_{T}^{2}\left\|f-f_{h}\right\|_{0 ; T}^{2}\right)^{\frac{1}{2}}\|\nabla v\|_{0 ; 0, \Omega \cup \Omega_{h}}
$$

and improve thereby the order of this term. But there is no way to compute this $L^{2}$-projection for general $f$ solely on the base of a given subroutine $x \mapsto f(x)$. But this is assumed to be the only way $f$ (and also $g^{\partial}$ ) enters the numerical algorithm.

ii. There is no limitation of the polynomial order of the finite element space. However, since we use a piecewise linear approximation of the boundary only, the corresponding error terms will not be of higher order if we use elements of degree more than 1.

iii. Theorem 2.ii shows that in case of negligible error in data $f$ and $g^{\partial}$ the error can be bounded, from both sides, in terms of the continuous residual $r\left(u_{h}\right)$ and the singular residual $\tilde{D}_{1}^{2}\left(u_{h}\right)$ on $\tilde{\mathcal{T}}_{h}$. In the general case Theorem 2. i such a result holds at least on $\mathcal{T}_{h}$ and this could be improved if one provides an appropriate definition of $\tilde{u}_{h}$.

Remark 3. In the following we sketch, for $n=2$, how to prove monotone decrease of the error for the adaptive algorithm. For details of most of the arguments see [Dö1, Dö2]. In Theorems 1 and 2 we proved estimates for the error $e_{h}$ on a grid $\mathcal{T}_{h}$ in terms of quantities depending on $u_{h}, f_{h}$ and the error in data. Let for $T \in \mathcal{T}_{h}$ and $\mathcal{A} \subseteq \mathcal{T}_{h}$

$$
\begin{aligned}
\eta_{T}^{2} & :=c_{1}^{2} d_{T} \sum_{E \in \mathcal{E}_{h ; T}^{\circ}}\left\|\left[\partial_{n} u_{h}\right]\right\|_{0 ; E}^{2}+c_{2}^{2} d_{T}^{2}\left\|\Delta u_{h}+f_{h}\right\|_{0 ; T}^{2}, \\
\eta_{\mathcal{A}}^{2} & :=\sum_{T \in \mathcal{A}} \eta_{T}^{2} .
\end{aligned}
$$

If the error reaches the saturated state, that is, if we can guarantee that from a certain grid $\mathcal{T}_{h_{0}}$ on, the error in data on $\mathcal{T}_{h}$ is smaller than $\mu_{0} \eta_{\mathcal{T}_{h}}$ (for sufficiently small $\mu_{0}$, depending on $\sigma_{h}$ and $M$ ), and $\Omega_{h}$ is in the saturated state for all refinements $\mathcal{T}_{h}$ of $\mathcal{T}_{h_{0}}$, we can prove that

$$
c \eta_{\mathcal{T}_{h}} \leq\left\|e_{h}\right\|_{0} \leq C \eta_{\mathcal{T}_{h}}
$$

with positive constants $c$ and $C$ depending on $\sigma_{h}$ and $M$. This will be the case if data $(f, g, \partial \Omega)$ is sufficiently smooth and approximated of higher order than the discretization error for the differential equation. A robust adaptive algorithm should in the beginning compute the local errors $\eta_{T}$ as well as the corresponding 
data error terms (see the right hand side of the inequality in Theorem 1). If there is evidence that the data error becomes negligible, its computation may be avoided. For proving convergence of the adaptive method, we have to be specific about the marking strategy and the refining strategy. Assume that we have computed the discrete solution on $\mathcal{T}_{H}$ and the local errors $\left(\eta_{T}\right)_{T \in \mathcal{T}_{H}}$. Now we choose a set $\mathcal{A}_{H} \subseteq \mathcal{T}_{H}$ such that

$$
\eta_{\mathcal{A}_{H}} \geq \vartheta \eta_{\mathcal{T}_{H}}
$$

where $\vartheta \in(0,1)$ is a given parameter (for more information about $\vartheta$ and $\mathcal{A}_{H}$ see [Dö1]). We now construct a refinement $\mathcal{T}_{h}$ of $\mathcal{T}_{H}$ in such a way that to each $T \in \mathcal{A}_{H}$ the local space $\left\{\psi_{h} \in \stackrel{\circ}{V}_{h} \mid \operatorname{supp}\left(\psi_{h}\right) \subseteq T\right\}$ is of sufficiently large dimension (depending on the polynomial degree of the local residual). This enables us to prove that $c \eta_{\mathcal{T}_{H}} \leq\left\|\nabla\left(u_{h}-u_{H}\right)\right\|_{0}$ for $\mu_{0}$ sufficiently small and $c$ depending on $\mu_{0}, \vartheta$, $\sigma_{h}$ and $M$ (similar to the proof of Theorem 2). Secondly, we have to establish an estimate of the form

$$
\left\|\nabla e_{H}\right\|_{0}^{2} \geq\left\|\nabla e_{h}\right\|_{0}^{2}+\frac{1}{2}\left\|\nabla\left(u_{H}-u_{h}\right)\right\|_{0}^{2}+C \mu_{0} \eta_{\mathcal{T}_{H}}^{2} .
$$

This proof is quite technical and uses, in addition, that Definition 3 is fulfilled with 2 replaced by $1+\gamma$, for sufficiently small positive $\gamma$ (depending on $\sigma$ and $M$ ). The proof, however, is significantly simplified if we assume that $\Omega$ is convex. With $\mu_{0}$ small enough, we will obtain from this

$$
\left\|\nabla e_{h}\right\|_{0} \leq \kappa\left\|\nabla e_{H}\right\|_{0}
$$

for some $\kappa \in(0,1)$. Note that such a result cannot hold on coarse grids [Dö1].

In the following we assume that $\Omega$ is convex. In this case we have that $\Omega_{h} \subseteq \Omega$ and

$$
\left\|\nabla^{2} v\right\|_{0 ; \Omega} \leq\|\Delta v\|_{0 ; \Omega} \quad \forall v \in H^{2,2}(\Omega) \cap \stackrel{\circ}{H}^{1,2}(\Omega)
$$

[Ka] (on non-convex domains with smooth boundary such an estimate would also hold but with a constant different from 1).

Theorem 3 ( $L^{2}$-a posteriori error estimates on convex domains). For $E \in \mathcal{E}_{h}^{\partial}$ let $B_{P}^{E}$ and $B_{\partial, 1}^{E}, B_{\partial, 2}^{E}$ be some constants for which the Sobolev estimates

$$
\|v\|_{0 ; \Omega^{E}} \leq B_{P}^{E}\left\|\nabla^{2} v\right\|_{0 ; \Omega^{E}}, \quad\|v\|_{0 ; \partial \Omega^{E} \cap \Omega} \leq B_{\partial, 1}^{E}\left\|\partial_{n} v\right\|_{0 ; E}+B_{\partial, 2}^{E}\left\|\partial_{n}^{2} v\right\|_{0 ; \Omega^{E}}
$$

( $n$ denotes the outer normal on $E$ ) hold for all $v \in H^{2,2}\left(\Omega^{E}\right)$ with $v \equiv 0$ on $\partial \Omega^{E} \cap$ $\partial \Omega$. Let $B_{\partial}^{E}:=C_{0}\left(\Omega_{h}\right) B_{\partial, 1}^{E}+B_{\partial, 2}^{E}$ for $C_{0}\left(\Omega_{h}\right):=C_{\partial}\left(\Omega_{h}\right)\left(1+d_{\Omega_{h}}^{-1} C_{P}(\Omega)\right)$. Now define $\tilde{\tilde{h}}$ as in Definition 1, but with $d_{T}$ replaced by $d_{T}^{2}$ and $C_{P}^{E}, C_{\partial}^{E} d_{E}^{-\frac{1}{2}}$ replaced by $B_{P}^{E}, B_{\partial}^{E} d_{E}^{-\frac{3}{2}}$, respectively. Define $\tilde{D}_{2}^{2}$ by replacing $\tilde{h}$ by $\tilde{\tilde{h}}$, and $\tilde{D}_{2, \partial}^{2}$ by replacing $C_{\partial}^{E}$ by $B_{\partial}^{E}$. Then, with constants $c_{3}, c_{4}$ depending on $\sigma_{h}, n$, and $M$ only,

$$
\begin{aligned}
\left\|e_{h}\right\|_{0 ; \Omega} \leq & c_{3}\left\|\tilde{\tilde{h}} r\left(u_{h}\right)\right\|_{\mathcal{T}_{h}}+c_{4} \tilde{D}_{2}^{2}\left(u_{h}\right)+\left\|\tilde{\tilde{h}} r\left(\tilde{u}_{h}\right)\right\|_{\tilde{\mathcal{T}}_{h} \backslash \mathcal{T}_{h}}+\tilde{D}_{2, \partial}^{2}\left(\tilde{u}_{h}\right) \\
& +2\left\|\tilde{g}-\tilde{g}_{h}\right\|_{0 ; \Omega}+C_{0}(\Omega)\left\|\tilde{g}-\tilde{g}_{h}\right\|_{0 ; \partial \Omega}+C_{P}(\Omega)^{2}\left\|f-f_{h}\right\|_{0 ; \Omega_{h}} .
\end{aligned}
$$

Proof. Let $\chi \in H^{2,2}(\Omega)$ be the weak solution of the problem

$$
-\Delta \chi=e_{h},\left.\quad \chi\right|_{\partial \Omega}=0 .
$$

$\chi$ satisfies the a priori estimates

$$
\|\nabla \chi\|_{0 ; \Omega} \leq C_{P}(\Omega)\left\|e_{h}\right\|_{0 ; \Omega}, \quad\left\|\nabla^{2} \chi\right\|_{0 ; \Omega} \leq\left\|e_{h}\right\|_{0 ; \Omega}
$$


Using the definition of $\chi, \Omega_{h} \backslash \Omega=\emptyset$ and performing the same operations as in the proof of Theorem 1 yields (since $e_{h}-\left(\tilde{g}-\tilde{g}_{h}\right) \in \stackrel{\circ}{H}^{1,2}(\Omega)$ )

$$
\begin{aligned}
\int_{\Omega}\left(e_{h}-\left(\tilde{g}-\tilde{g}_{h}\right)\right) e_{h}= & \int_{\Omega} \nabla\left(e_{h}-\left(\tilde{g}-\tilde{g}_{h}\right)\right) \cdot \nabla \chi \\
= & \left(r\left(\tilde{u}_{h}\right), \chi-\chi_{h}\right)_{\tilde{\mathcal{T}}_{h}}+\left(\left[\partial_{h} \tilde{u}_{h}\right], \chi-\chi_{h}\right)_{\tilde{\mathcal{E}}_{h}} \\
& +\int_{\Omega_{h}}\left(f-f_{h}\right) \chi-\int_{\partial \Omega}\left(\tilde{g}-\tilde{g}_{h}\right) \partial_{h} \chi+\int_{\Omega}\left(\tilde{g}-\tilde{g}_{h}\right) \Delta \chi
\end{aligned}
$$

for arbitrary $\chi_{h} \in \stackrel{\circ}{V}_{h}$. Now take $\chi_{h}:=I_{h}^{0} \chi$. Note that for $I_{h}$, as defined in Section 3, it was proved in $[\mathrm{Cl}]$ that $\left\|\chi-I_{h} \chi\right\|_{0 ; T} \leq c d_{T}^{2}\left\|\nabla^{2} \chi\right\|_{0 ; \omega_{T}}$ for all $T \in \mathcal{T}_{h}$. Performing the same computations as in the proof of Lemma 2 we obtain for $r\left(\tilde{u}_{h}\right) \in L^{2}\left(\tilde{\mathcal{T}}_{h}\right)$

$$
\begin{aligned}
& \left|\left(r\left(\tilde{u}_{h}\right), \chi-I_{h}^{0} \chi\right)_{\tilde{\mathcal{T}}_{h}}\right| \\
& \quad \leq c\left\|\tilde{\tilde{h}} r\left(\tilde{u}_{h}\right)\right\|_{\mathcal{T}_{h}}\left\|\nabla^{2} \chi\right\|_{0 ; \Omega}+\sum_{E \in \mathcal{E}_{h}^{\partial}}\left\|r\left(\tilde{u}_{h}\right)\right\|_{0 ; \Omega^{E}}\|\chi\|_{0 ; \Omega^{E}} \\
& \quad+c \sum_{T \in \mathcal{T}_{h}^{\partial}} d_{T}^{2}\left\|r\left(\tilde{u}_{h}\right)\right\|_{0 ; T}\left(\left\|\nabla^{2} \chi\right\|_{0 ; \omega_{T}}+\sum_{p \in \mathcal{N}_{h ; T}^{\partial}} \min _{E \in \mathcal{E}_{h ; p}^{\partial}} d_{E}^{-\frac{3}{2}}\|\chi\|_{0 ; E}\right) .
\end{aligned}
$$

Inserting the Sobolev inequality into the second term, it is estimated by

$$
\left(\sum_{E \in \mathcal{E}_{h}^{\partial}} B_{P}^{E^{2}}\left\|r\left(\tilde{u}_{h}\right)\right\|_{0 ; \Omega^{E}}^{2}\right)^{\frac{1}{2}}\left\|\nabla^{2} \chi\right\|_{0 ; \Omega \backslash \Omega_{h}},
$$

whereas the fourth term can be estimated using

$$
\begin{aligned}
\sum_{T \in \mathcal{T}_{h}^{\partial}} d_{T}^{2}\left(\sum_{p \in \mathcal{N}_{h ; T}^{\partial}} \min _{E \in \mathcal{E}_{h ; p}^{\partial}} d_{E}^{-\frac{3}{2}}\left(B_{\partial, 1}^{E}\left\|\partial_{n} \chi\right\|_{0 ; E}+B_{\partial, 2}^{E}\left\|\partial_{n}^{2} \chi\right\|_{0 ; \Omega^{E}}\right)\right)\left\|r\left(u_{h}\right)\right\|_{0 ; T} \\
\leq c \sum_{T \in \mathcal{T}_{h}^{\partial}} d_{T}^{2}\left(\sum_{p \in \mathcal{N}_{h ; T}^{\partial}} \min _{E \in \mathcal{E}_{h ; p}^{\partial}} d_{E}^{-\frac{3}{2}}\left(C_{0}\left(\Omega_{h}\right) B_{\partial, 1}^{E}+B_{\partial, 2}^{E}\right)\right) \\
\times\left(\sum_{E \in \mathcal{E}_{h ; T^{\prime}}^{\partial}: T^{\prime} \in \omega_{T}} C_{0}\left(\Omega_{h}\right)^{-1}\left\|\partial_{n} \chi\right\|_{0 ; E}+\left\|\partial_{n}^{2} \chi\right\|_{0 ; \Omega^{E}}\right)\left\|r\left(u_{h}\right)\right\|_{0 ; T} \\
\leq c\left(\sum_{T \in \mathcal{T}_{h}^{\partial}} d_{T}^{4}\left(\sum_{p \in \mathcal{N}_{h ; T}^{\partial}} \min _{E \in \mathcal{E}_{h ; p}^{\partial}} d_{E}^{-\frac{3}{2}} B_{\partial}^{E}\right)^{2}\left\|r\left(u_{h}\right)\right\|_{0 ; T}^{2}\right)^{\frac{1}{2}} \\
\times\left(C_{0}\left(\Omega_{h}\right)^{-1}\left\|\partial_{n} \chi\right\|_{0 ; \partial \Omega_{h}}+\left\|\nabla^{2} \chi\right\|_{0 ; \Omega \backslash \Omega_{h}}\right) .
\end{aligned}
$$

Using the trace inequality from Lemma 1 applied to $\nabla \chi$ and the a priori estimates for $\chi$ yields

$$
\begin{aligned}
\left\|\partial_{n} \chi\right\|_{0 ; \partial \Omega_{h}} & \leq C_{\partial}\left(\Omega_{h}\right)\left(\left\|\nabla^{2} \chi\right\|_{0 ; \Omega_{h}}+d_{\Omega_{h}}^{-1}\|\nabla \chi\|_{0 ; \Omega_{h}}\right) \\
& \leq C_{\partial}\left(\Omega_{h}\right)\left(1+d_{\Omega_{h}}^{-1} C_{P}(\Omega)\right)\left\|e_{h}\right\|_{0 ; \Omega}=C_{0}\left(\Omega_{h}\right)\left\|e_{h}\right\|_{0 ; \Omega}
\end{aligned}
$$

and we finally obtain

$$
\left|\left(r\left(u_{h}\right), \chi-\chi_{h}\right)_{\mathcal{T}_{h}}+\left(r\left(\tilde{u}_{h}\right), \chi\right)_{\tilde{\mathcal{T}}_{h} \backslash \mathcal{T}_{h}}\right| \leq\left(c\left\|\tilde{\tilde{h}} r\left(u_{h}\right)\right\|_{\mathcal{T}_{h}}+\|\left.\tilde{\tilde{h}} r\left(\tilde{u}_{h}\right)\right|_{\tilde{\mathcal{T}}_{h} \backslash \mathcal{T}_{h}}\right)\left\|e_{h}\right\|_{0 ; \Omega} .
$$

The estimate for $\left|\left(\left[\partial_{n} u_{h}\right], \chi-\chi_{h}\right)_{\tilde{\mathcal{E}}_{h}}\right|$ follows in the same way. 
Remark 4. $i$. The constants $c_{1}, \ldots, c_{4}$ from Theorems 1 and 3 are of moderate size. To fix them in the numerical algorithm, one can compute some examples with known solutions and compare the sizes of the different error terms and then adjust factors such that the computed error is some factor larger than the exact error. This is what we have done in our implementation (see Section 7). In case $M>1$ we let $c_{1}=c_{2}, c_{3}=c_{4}$.

$i$. To prove Theorem 3 for non-convex domains with smooth boundary one needs a $H^{2,2}$-continuous extension of $\chi$ to $\Omega \cup \Omega_{h}$ and one would get additional error terms on $\Omega_{h} \backslash \Omega$. Note that in the derivation of Theorem 1 we make use of the fact that $v \in \stackrel{\circ}{H}^{1,2}(\Omega)$ could be extended $H^{1,2}$-continuously by zero.

iii. By scaling we see that $C_{\partial}\left(\Omega_{h}\right)$ behaves like $O\left(d_{\Omega}^{\frac{1}{2}}\right)$. Unfortunately we have no further estimates in terms of simple available geometric constants. In case $n=2$ estimates for $B_{\partial, 1}^{E}, B_{\partial, 2}^{E}$, and $B_{P}^{E}$ are provided in Section 6 .

$i v$. The main task in the preceding estimate was to ensure that the additional terms, compared to the standard interpolation estimate, did not get dominant if the grid size tends to zero. Therefore the term $\|\chi\|_{0 ; E}$ has to be estimated very carefully and in the interpolation estimate we cannot argue only locally in analogy to the proof of Theorem 1 . To ensure that $\tilde{\tilde{h}}$ behaves like $d_{T}^{2}$ (for $T \in \mathcal{T}_{h}^{\partial}$ ) up to higher order terms for $d_{T} \rightarrow 0$ we would otherwise need an estimate $\|\chi\|_{0 ; E} \leq B_{\partial}^{E}\left\|\nabla^{2} \chi\right\|_{0 ; \Omega^{E}}$, with $B_{\partial}^{E} \leq C d_{E}^{\frac{3}{2}}$. (We note here, without proof, that if $\Omega$ is convex and polygonally bounded, such an estimate can be established.) But in general we only obtain $B_{\partial}^{E}=O\left(d_{E}\right)$ as can be seen by the following example:

$$
\begin{aligned}
& \Omega^{E}=\left\{(s, t) \mid 0 \leq t \leq\left(d^{2}-s^{2}\right)\right\}, \\
& \chi(s, t)=t-\left(d^{2}-s^{2}\right) \\
& \text { so that }\left\|\nabla^{2} \chi\right\|_{0 ; \Omega_{E}}=4 / \sqrt{3} d^{\frac{3}{2}} \text { and }\|\chi\|_{0 ; E}=4 / \sqrt{15} d^{\frac{5}{2}} .
\end{aligned}
$$

\section{Estimates for Sobolev constants}

In this section we will give estimates for $C_{P}^{E}, C_{\partial}^{E}, B_{\partial, 1}^{E}, B_{\partial, 2}^{E}$, and $B_{P}^{E}$ in the two-dimensional case. In three dimensions further knowledge on the shape of $\Omega^{E}$ is necessary to proceed similarly.

Let $R_{E}$ be a rectangle containing $\Omega^{E}$ with edges parallel and orthogonal to $E$. $R_{E}$ can be decomposed in the form $R_{E}=R_{E}^{(1)} \cup \tilde{E} \cup R_{E}^{(2)}$, where $\tilde{E}$ is the intersection between $\Omega^{E}$ and the continuation of $E$ and $R_{E}^{(1)}, R_{E}^{(2)}$ are the connected components of $R_{E} \backslash \tilde{E}$. Then we immediately see that $C_{P}^{E} \leq \max \left\{C_{P}^{(1)}, C_{P}^{(2)}\right\}$, where $C_{P}^{(i)}:=$ $C_{P}\left(R_{E}^{(i)}, \partial R_{E}^{(i)} \backslash \tilde{E}\right)$. To estimate each $C_{P}^{(i)}$ we assume without loss of generality that $\tilde{E}:=[0, L], R_{E}^{(i)}:=[0, L] \times[0, H]$ and extend $v$ by 0 outside $\Omega^{E}$. Substituting $v(s, t)$ for $(s, t) \in[0, L] \times[0, H]$ by $v(s, H)-\int_{t}^{H} \partial_{2} v\left(s, t^{\prime}\right) d t^{\prime}$ we easily obtain the estimates $\|v\|_{0 ;[0, L] \times[0, H]} \leq H\|\nabla v\|_{0 ;[0, L] \times[0, H]}$ and $\|v\|_{0 ; E} \leq H^{\frac{1}{2}}\|\nabla v\|_{0 ;[0, L] \times[0, H]}$; thus $C_{P}^{(i)} \leq H$ and $C_{\partial}^{E} \leq H^{\frac{1}{2}}$.

Asking for estimates for $B_{\partial, 1}^{E}, B_{\partial, 2}^{E}, B_{P}^{E}$ we assume as before $E \subset \mathbb{R} \times\{0\}$ and $\Omega^{E} \subset R:=[0, L] \times[0, H]$ because $\Omega$ is supposed to be convex. Furthermore, let $v \in H^{2,2}\left(\Omega^{E}\right)$ and let $\partial \Omega^{E} \backslash E$ be a piecewise smooth curve. For each $s \in E$ there is 
a closed interval $I_{s}=\left[0, h_{s}\right]$, for some $h_{s} \in[0, H]$, such that $v\left(s, h_{s}\right)=0$ and thus

$$
|v(s, 0)|=\left|\int_{I_{s}} \partial_{2} v\left(s, t^{\prime}\right) d t^{\prime}\right| \leq H\left|\partial_{2} v(s, 0)\right|+H^{\frac{3}{2}}\left\|\partial_{2}^{2} v(s, .)\right\|_{0 ; I_{s}} .
$$

Taking the square and integrating yields $\|v\|_{0 ; E} \leq \sqrt{2}\left(H\left\|\partial_{2} v\right\|_{0 ; E}+H^{\frac{3}{2}}\left\|\partial_{2}^{2} v\right\|_{0 ; \Omega^{E}}\right)$, so that $B_{\partial, 1}^{E} \leq \sqrt{2} H, B_{\partial, 2}^{E} \leq \sqrt{2} H^{\frac{3}{2}}$. Finally, let $J_{t}:=\left\{(s, t) \mid(s, t) \in \Omega^{E}\right\}$ for $t \in[0, H]$. Using $\left.v\right|_{\partial J_{t}}=0$ and $\int_{J_{t}} \partial_{1} v(s, t) d s=0$ we obtain $|v(s, t)|^{2} \leq$ $L^{3}\left\|\partial_{1}^{2} v(., t)\right\|_{0 ; J_{t}}^{2}$. Integrating over $\Omega^{E}$ we end up with $\|v\|_{0 ; \Omega^{E}} \leq L^{2}\left\|\nabla^{2} v\right\|_{0 ; \Omega^{E}}$; that is $B_{P}^{E} \leq L^{2}$.

If the grid size $h$ tends to 0 , it is reasonable to assume that $H \leq C d_{E}^{2}$ and $L=d_{E}$. Thereby $C_{P}^{E}, B_{P}^{E} \leq C d_{E}^{2}, C_{\partial}^{E} \leq C d_{E}$, and $B_{\partial}^{E} \leq C d_{E}^{2}$. This ensures that the effect of the boundary approximation on the error is of higher order for linear Finite Elements (see Theorems 1 and 3).

\section{The Algorithm}

In this section we discuss a robust implementation of linear Finite Elements for Poisson's equation on two-dimensional domains with piecewise smooth boundary. We especially take into account that only parts of the domain have to be triangulated and that data $f$ and $g$ can only be evaluated pointwise in $\Omega$ or on $\partial \Omega$, respectively. Furthermore, the different error terms have to be calculated efficiently. This means for the error terms due to the boundary approximation that the cost in storage and speed is proportional to the geometric complexity of the domains boundary. Therefore, it turns out to be less expensive than an overall equally fine approximation of $\Omega$ by $\Omega_{h}$.

At first, the a posteriori estimates for the energy norm given in Section 5 refer to the boundary $\partial \Omega$ of the continuous problem. The only restriction we made is that this boundary is piecewise smooth. However, we are unable to handle the exact boundary $\partial \Omega$ algorithmically. Instead we represent $\partial \Omega$ by some polygonal approximation $\mathcal{B}$. The initial boundary approximation is generated by a stable path following algorithm, which we have implemented for piecewise implicitly defined boundary segments. Thereby we obtain a sufficiently fine geometrical approximation. Before solving the first discrete problem we have to define some initial triangulation $\mathcal{T}_{h}$. We only assume boundary vertices of $\mathcal{T}_{h}$ to be (a subset of) points in $\mathcal{B}$. During the calculation, $\mathcal{T}_{h}$ is successively refined and in the course of this, we first refine $\mathcal{B}$ if necessary and then pick up points in $\mathcal{B}$ to refine the boundary of the triangulation. No assumption is made on a geometrically fitting shape and the initial mesh may cover only part of the domain. Therefore the algorithm has to be able to enlarge the discretized domain substantially if indicated by the a posteriori estimates. Under these circumstances a Delaunay technique to adapt the triangulation seems to be more preferable for refinement than strategies based on subdivision with boundary fitting [Bä, Ri]. For reference we cite [GH, We, Re]. We apply a Delaunay refinement based on point insertion and edge swapping as the basic operations. The local grid size is described by a function $h$ on all nodes $p \in \mathcal{N}_{\mathcal{T}_{h}}$. A subscript $h$ from now on indicates the corresponding fineness. Based on estimates of the local errors, the values of $h$ are locally decreased, and the refinement procedure then adapts the mesh until the desired grid size is again attained. The proposed adaptive algorithm can be summarized as follows: 


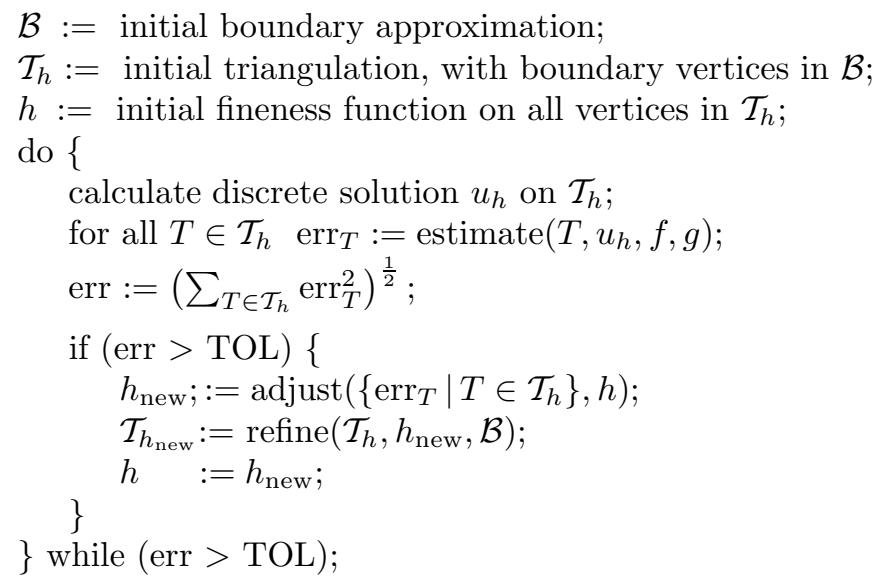

Subsequently, we explain the estimate, adjust and refine procedures, which deal with the concrete calculation of the a posteriori estimates, the marking of regions for refinement, and the actual mesh adaptation.

Estimating the error. The purpose of this section is to describe how to compute (an approximation of) the $H^{1,2}$-a posteriori error, that is, the left hand side of the assertion stated in Theorem 1. The "standard" term of this error estimate, the sum of the local element and edge residuals for $T \in \mathcal{T}_{h}$

$$
\eta_{T}^{2}:=c_{1}\left\|\tilde{h} f_{h}\right\|_{0 ; T}^{2}+c_{2} d_{T}^{-1} \tilde{h}_{T}^{2}\left\|\left[\partial_{n} u_{h}\right]\right\|_{0 ; \mathcal{E}_{h ; T}^{\circ}}^{2}+\sum_{E \in \mathcal{E}_{h ; T}^{\partial}} C_{\partial}^{E^{2}}\left\|\left[\partial_{n} u_{h}\right]\right\|_{0 ; \partial \Omega^{E} \cap \Omega}^{2}
$$

is easy to evaluate. For reliable handling of the data error $\left\|f-f_{h}\right\|_{0 ; \Omega \cap \Omega_{h}}$ we refer to [Dö1]. What is left in the case of linear Finite Elements is to argue about how to evaluate

$$
\left\|\nabla\left(\tilde{g}-\tilde{g}_{h}\right)\right\|_{0 ; \Omega}, \quad\|\tilde{h} f\|_{0 ; \tilde{\mathcal{T}}_{h} \backslash \mathcal{T}_{h}} .
$$

At first, we have to define the extensions $\tilde{g}$ and $\tilde{g}_{h}$. For $\tilde{g}_{h}$ we simply take $\tilde{g}_{h}=$ $\tilde{u}_{h}$, where $\tilde{u}_{h}$ on $\Omega^{E}$ is defined as the extension of the linear polynomial $\left.u_{h}\right|_{T(E)}$. Furthermore, on $\mathcal{T}_{h} \backslash \mathcal{T}_{h}^{\partial}$ we set $\tilde{g}=\tilde{g}_{h}$ and in the remaining boundary region we suppose that some interpolation within $\partial \Omega$ and the boundary of $\bigcup_{T \in \mathcal{T}_{h} \backslash \mathcal{T}_{h}{ }^{\partial}} T$ is given. To simplify the presentation, we assume that a segment of $\partial \Omega$ in between two boundary vertices intersects at most the interior of one element, and that boundary elements have only one boundary edge. The other cases can be treated similarly. For $E \in \mathcal{E}_{h}^{\partial}$ we denote $\tilde{\omega}_{E}=T(E) \cup \Omega^{E}$. Then it remains to consider

$$
\operatorname{err}_{g}\left(\tilde{\omega}_{E}\right):=\left\|\nabla\left(\tilde{g}-\tilde{g}_{h}\right)\right\|_{0 ; \tilde{\omega}_{E}}^{2} \text { and } \operatorname{err}_{f}\left(\Omega^{E}\right):=\|\tilde{h} f\|_{0 ; \Omega^{E}}^{2} .
$$

Now the basic idea is to replace the original estimates with computable and reliable formulas, for simplicity again denoted by $\operatorname{err}_{g}\left(\tilde{\omega}_{E}\right), \operatorname{err}_{f}\left(\Omega^{E}\right)$, using the polygonal boundary representation. Here we especially focus on an efficient and robust calculation, which means that the estimation procedure returns an upper bound of the true estimate; in the saturated case the calculated estimates should converge for an increasing computational resolution (see below) to the true values, and finally the calculation has to be fast.

At first we extract from the polygonal boundary $\mathcal{B}$ the segment in between the vertices of the boundary edge $E$. Then we temporarily insert for each line 

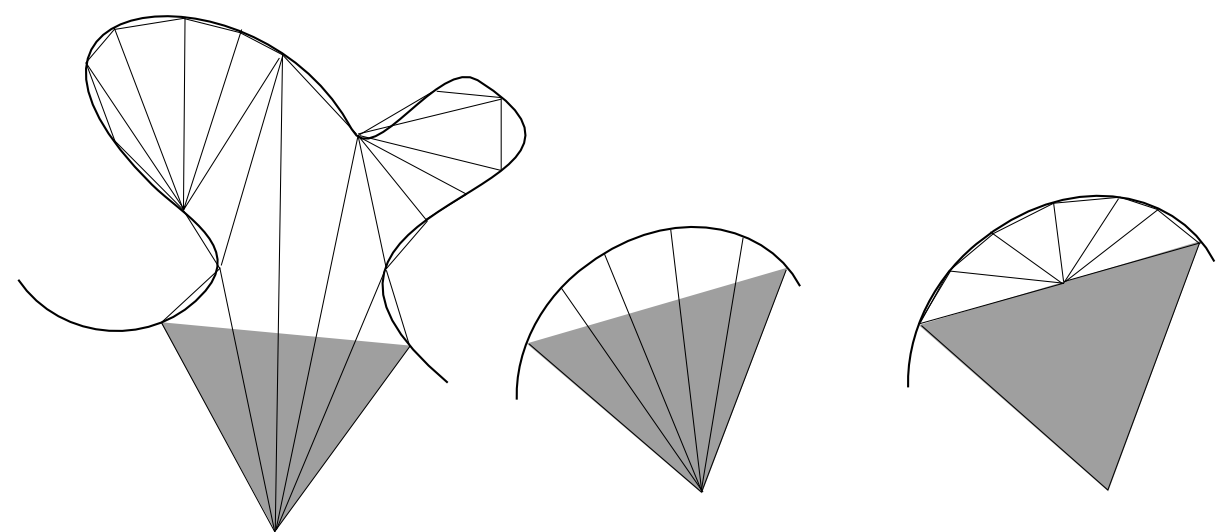

Figure 2. On the left the recursive subdivision of $\tilde{\omega}_{E}$ required in the calculation of $\operatorname{err}_{g}\left(\tilde{\omega}_{E}\right)$ is sketched in two cases. On the right we show an example of a triangle fan used to approximate $\operatorname{err}_{f}\left(\Omega^{E}\right)$. The true boundary $\partial \Omega$ is indicated by thick lines and the corresponding boundary triangles are shaded.

segment in $\mathcal{B}$ a fixed number of additional test points, randomly distributed on the corresponding segment of $\partial \Omega$. Inserting more test points will increase the quality of the evaluation. To do this, we again apply the path following algorithm already used to generate the initial boundary approximation. Now we have an improved representation of the boundary segment $\partial \tilde{\omega}_{E} \cap \partial \Omega$ at hand and we start to estimate the error, corresponding to the Dirichlet data $g$. We replace $\tilde{g}$ by the piecewise linear interpolation on the current refined polygonal boundary, which attains the values of $\tilde{g}$ at the vertices. Then we ask for an extension of this interpolated function onto the polygonally approximated domain $\tilde{\omega}_{E}$, for simplicity again called $\tilde{\omega}_{E}$. Therefore we temporarily mesh $\tilde{\omega}_{E}$ with triangles $\Delta_{i}$ for $0 \leq i \leq m$ spanned by vertices from the bounding polygon. On each of these triangles the extension is defined as the linear interpolation $I_{i} g$ of the true values of $\tilde{g}$ at the vertices. In the saturated case, where $\tilde{\omega}_{E}$ is convex, a fan-like mesh appears to be the canonical choice (Figure 2). In the general case we proceed similarly. First we build a fan of triangles contained in $\tilde{\omega}_{E}$ and centered at the unique vertex from $\partial \tilde{\omega}_{E}$ not located on $\partial \Omega$. Then we mesh the remaining "bubbles" by a recursive call of the fan generation (Figure 2). Finally we compute

$$
\operatorname{err}_{g}\left(\tilde{\omega}_{E}\right)=\sum_{0 \leq i \leq m} \int_{\Delta_{i}}\left|\nabla\left(I_{i} \tilde{g}-\tilde{g}_{h}\right)\right|^{2}
$$

Next, we give a formula to calculate $\operatorname{err}_{f}\left(\Omega^{E}\right)$. This is again based on the same temporarily refined polygonal boundary segment. Now we construct a fan of triangles $\Delta_{j}$ for $0 \leq j \leq r$ centered at the midpoint of the boundary edge and covering the region in between the edge and the boundary polygon (Figure 2) In the non-saturated case this covering might overlap. Let us denote by $h_{\Omega^{E}}^{\perp}$ the diameter of $\Omega^{E}$ in the direction normal to $E$. Then we evaluate

$$
\operatorname{err}_{f}\left(\Omega^{E}\right)=\sum_{0 \leq j \leq r}\left(h_{\Omega^{E}}^{\perp}\right)^{2} \chi_{\Omega \backslash \Omega_{h}}\left(y_{j}\right) f\left(y_{j}\right)^{2}\left|\Delta_{j}\right|,
$$



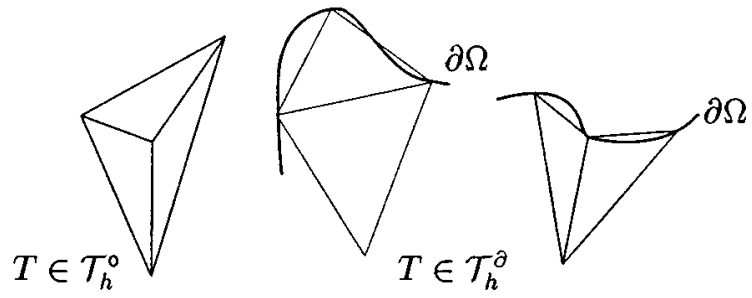

FiguRE 3. Three different refinement operations on triangles.

where $y_{j}$ is a random point in $\Delta_{j}$ and $\chi_{A}$ is the indicator function of a set $A$. In the general case an overlap in the covering would lead to an overestimate of the error, whereas in the saturated case a sufficient approximation of the exact value is obtained.

If we increase the number of test points inserted temporarily into the boundary list we improve the reliable calculation of both estimates $\operatorname{err}_{g}\left(\tilde{\omega}_{E}\right)$ and $\operatorname{err}_{f}\left(\Omega^{E}\right)$, despite only one test point per triangle $\Delta_{j}$ for the $f$ evaluation.

Let us finally collect the different local error terms in the following definition of a local error estimator $\operatorname{err}_{T}$ :

$$
\operatorname{err}_{T}^{2}:=\eta_{T}^{2}+C_{P}(\Omega)\left\|f-f_{h}\right\|_{0 ; T \cap \Omega}^{2}+\operatorname{err}_{f}\left(\Omega^{E}\right)+2 \operatorname{err}_{g}\left(\tilde{\omega}_{E}\right)
$$

for an element $T \in \mathcal{T}_{h}$ with boundary edge $\mathcal{E} \in \mathcal{E}_{h}^{\partial}$, which is used in the above algorithm.

Refining the triangulation. The desired grid size $h$ on the vertices of the current $T$ is adjusted following each error estimation in the algorithm; therefore, we mark triangles for refinement. Then we multiply the $h$ values on vertices of marked triangles by some refinement factor $\beta<1$ ( $\beta=\frac{1}{2}$ in the above examples) which describes the desired decrease in the local grid size. Afterward, we successively refine the triangulation (see below) until the prescribed fineness $h$ is attained on all triangles. Here the refinement of a single triangle is the basic operation. Each single triangle fulfills the refinement criteria corresponding to the function $h$ if $d_{T}<h(p) \forall p \in \mathcal{N}_{T}$. Interior triangles $T \in \mathcal{T}_{h}^{\circ}$ are refined by inserting their bary center as a new point; boundary triangles $T \in \mathcal{T}_{h}^{\partial}$ with the boundary edge as longest edge are refined adding a new point from the boundary polygon $\mathcal{B}$. If there is none on the corresponding segment of $\partial \Omega$, an additional point is in advance added to $\mathcal{B}$ (Figure 3). In any case afterward we start a recursive swap operation on pairs of the new created triangles and the adjacent elements. This ensures that we again obtain a Delaunay triangulation [Re]. The values of $h$ on the new vertices are defined as linear interpolation of the $h$ values on the vertices of the refined edge or the refined triangle, respectively. Following this refinement strategy we implicitly assume that no overlaps will occur. Otherwise the proposed meshing process has to be replaced by some global Delaunay remeshing, which is out of the scope of the presented work (see below the remarks on the 3D case). In the applications we have studied and for the examples given in this paper this assumption has always been fulfilled.

Preconditioning the linear solver. The Delaunay refinement procedure supports us with a hierarchy of grids. In contrast to other refinement algorithms here 
the corresponding function spaces are not nested. But nevertheless we can implement a hierarchical preconditioning $[\mathrm{Y}]$ where, depending on the type of triangle refinement, each node except those on the initial triangulation has 2 or 3 parent nodes with weights $\frac{1}{2}$ and $\frac{1}{3}$, respectively. In the examples this preconditioning turns out to be efficient. In the next section a table gives results for a specific application. Therein, the last column indicates the moderate increase of necessary CG iterations for a rapidly increasing number of unknowns.

Remark on the three-dimensional case. In the preceding paragraphs we have discussed a two-dimensional implementation which efficiently takes into account the effects of a posteriori measured error terms due to a possibly coarse boundary approximation. The same ideas carry over to three dimensions although a lot of programming effort would be necessary. For three-dimensional domains with piecewise smooth boundaries Delaunay techniques, which create an environmental mesh including the actual domain and all inside holes, have turned out to be appropriate $[\mathrm{Ba}, \mathrm{HTH}]$. An additional constraint in the mesh generation, that faces on $\partial \Omega$ are represented by element faces, then allows the extraction of a mesh for $\Omega_{h}$. Typically, very few outer points are sufficient to create such a mesh, which furthermore involves a natural segmentation of $\Omega \backslash \Omega_{h}$ into sets $\Omega^{E}$ associated to boundary faces $E \in \mathcal{E}_{h}^{\partial}$, which is required by the error analysis presented.

\section{NumericAL EXAMPLES}

In this section we give some examples which underline the capabilities of the algorithm discussed.

At first we compare the order of the different error terms in our estimate for a domain with smooth boundary. The singular part (sing.) consisting of the jumps of the normal derivatives on the inner edges and the element residual (el.res.) are of order $O(h)$, where the error terms $\left\|\nabla\left(\tilde{g}-\tilde{g}_{h}\right)\right\|_{0 ; \Omega},\|\tilde{h} f\|_{0 ; \tilde{\mathcal{T}}_{h} \backslash \mathcal{T}_{h}}$ are of order $O\left(h^{\frac{3}{2}}\right)$ and $O\left(h^{3}\right)$, respectively. Finally, the total estimated error (err) and the relative error (rel.err.) are listed. The table below lists all these values for a sequence of uniform grid sizes $h$ and for $\Omega=B_{1}(0), f=1$ and $g=2 x^{2}-1$.

\begin{tabular}{|l|l|l|l|l|l|l|r|r|}
\hline$h$ & sing. & el.res. & $\operatorname{err}_{g}$ & err $_{f}$ & err & rel.e. & $\sharp \mathcal{N}_{\mathcal{T}_{h}}$ & $\sharp$ it \\
\hline \hline 1.0 & 0.000 & $6.46 \mathrm{e}-1$ & 3.935 & $8.03 \mathrm{e}-1$ & 7.996 & 6.793 & 3 & 0 \\
$5.0 \mathrm{e}-1$ & $3.71 \mathrm{e}-1$ & $2.07 \mathrm{e}-1$ & $1.00 \mathrm{e}-1$ & $3.73-\mathrm{e} 3$ & $5.50 \mathrm{e}-1$ & $2.15 \mathrm{e}-1$ & 47 & 11 \\
$2.5 \mathrm{e}-1$ & $1.98 \mathrm{e}-1$ & $1.11 \mathrm{e}-1$ & $9.79 \mathrm{e}-2$ & $1.10 \mathrm{e}-3$ & $2.80 \mathrm{e}-1$ & $1.09 \mathrm{e}-1$ & 129 & 13 \\
$1.3 \mathrm{e}-1$ & $1.02 \mathrm{e}-1$ & $5.64 \mathrm{e}-2$ & $2.63 \mathrm{e}-2$ & $1.38 \mathrm{e}-4$ & $1.15 \mathrm{e}-1$ & $4.44 \mathrm{e}-2$ & 475 & 17 \\
$6.3 \mathrm{e}-2$ & $5.07 \mathrm{e}-2$ & $2.82 \mathrm{e}-2$ & $9.81 \mathrm{e}-3$ & $1.67 \mathrm{e}-5$ & $5.44 \mathrm{e}-2$ & $2.11 \mathrm{e}-2$ & 1792 & 20 \\
$3.1 \mathrm{e}-2$ & $2.57 \mathrm{e}-2$ & $1.42 \mathrm{e}-2$ & $3.62 \mathrm{e}-3$ & $2.53 \mathrm{e}-6$ & $2.67 \mathrm{e}-2$ & $1.03 \mathrm{e}-2$ & 6979 & 25 \\
$1.6 \mathrm{e}-2$ & $1.26 \mathrm{e}-2$ & $6.99 \mathrm{e}-3$ & $1.34 \mathrm{e}-3$ & $3.52 \mathrm{e}-7$ & $1.29 \mathrm{e}-2$ & $4.99 \mathrm{e}-3$ & 28378 & 26 \\
$7.8 \mathrm{e}-3$ & $6.33 \mathrm{e}-3$ & $3.51 \mathrm{e}-3$ & $4.45 \mathrm{e}-4$ & $3.11 \mathrm{e}-8$ & $6.39 \mathrm{e}-3$ & $2.48 \mathrm{e}-3$ & 111152 & 29 \\
\hline
\end{tabular}

The following figures show results obtained for various applications. They especially emphasize that contributions to the error from outside the initial grid are detected (see Figures 4, 5, 6). Furthermore, the algorithm resolves geometrical singularities not in advance but depending on the local error estimates (see some of the cusps in Figure 4, and the corner in Figure 5). Finally, parts of the domain 

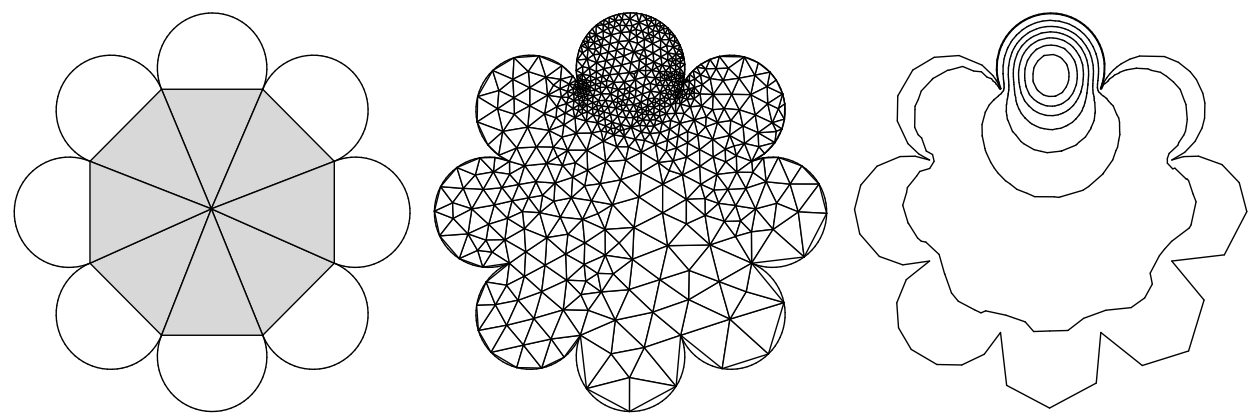

Figure 4. Given a domain with cusps and $g=0, f=\chi(A)$ where $A$ is a small ball inside one of the non-discretized "bubbles" of the initial grid, the initial and the final grid and isolines of the solution on the final discretization are shown.
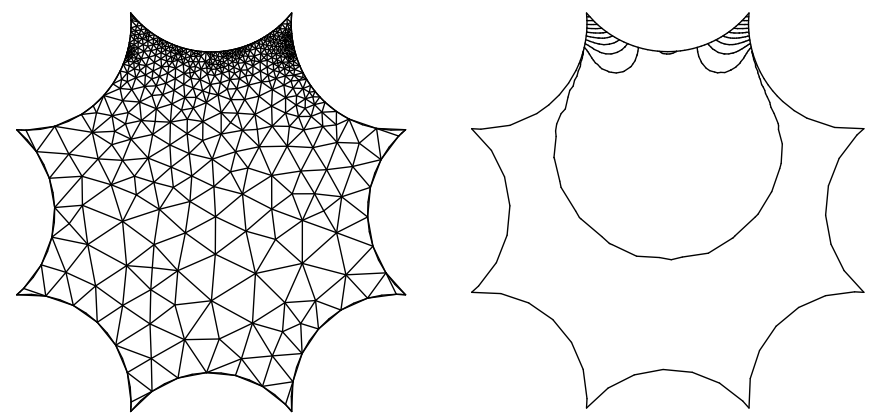

Figure 5. Given a star shaped domain and $f=0, g=$ $\max (0, y-C)$, the final grid and isolines of the solution are drawn.
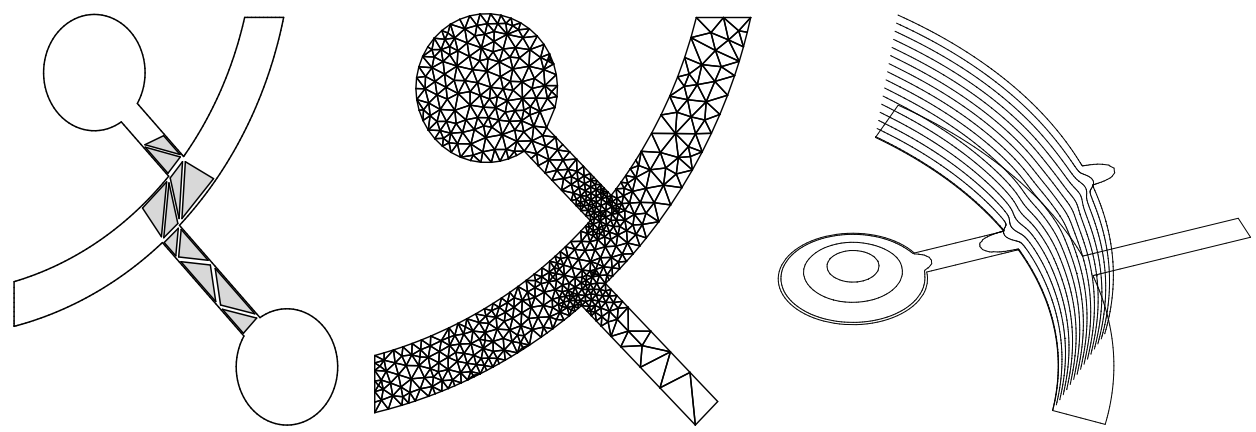

Figure 6. A coarse starting grid inside a complex domain, the final adaptive finite element mesh, and a perspective view on an isoline image of the solution.

with very small error contributions will stay non-discretized (see one of the circular regions in Figure 6). Figure 7 shows the behavior of our algorithm at corner points of $\partial \Omega$ that are not vertex of the triangulation. 

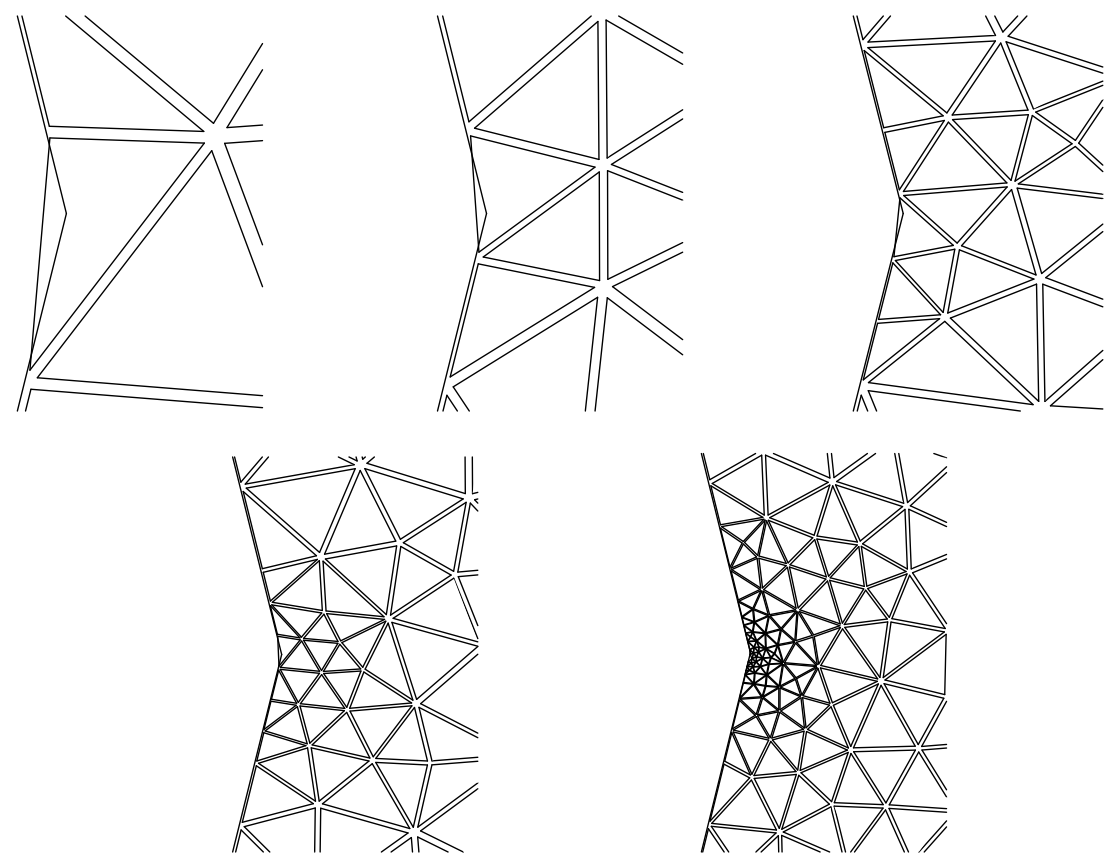

FiguRE 7. Successive mesh refinement steps at a domain corner, where the corner point is explicitly not depicted by the algorithm. This case is not covered by our theory. Nevertheless the algorithms also work robustly in this case.

\section{REFERENCES}

[Ad] Adams, R. A. (1975): Sobolev spaces. Academic Press, New York. MR 56:9247

[Al] Alt, H. W. (1985): Lineare Funktionalanalysis. Springer, Berlin.

[Bä] Bänsch, E. (1991): Local mesh refinement in 2 and 3 dimensions. Impact Comput. Sci. Engrg., 3, 181-191. MR 92h:65150

[BK] Bramble, J. H., King, J. T. (1994): A robust finite element method for non-homogeneous Dirichlet problems in domains with curved boundaries. Math. Comp., 63, 1-17. MR 94i:65112

[BR] Babuška, I., Rheinboldt, W. C. (1978): Error estimators for adaptive finite element computations. SIAM J. Numer. Anal., 15, 736-754. MR 58:3400

[BW] Bank, R. E., Weiser, A. (1985): Some a priori error estimators for elliptic partial differential equations. Math. Comp., 44, 283-301. MR 86g:65207

[Ba] Baker, T. J. (1989): Automatic mesh generation for complex three-dimensional regions using a constrained Delaunay triangulation. Engineering with Computers, 5, 161-175.

[Ci] Ciarlet, P. G. (1978): The finite element method for elliptic problems. Studies in Mathematics and its Applications, North-Holland, Amsterdam, 2. Edition. MR 58:25001

[Cl] Clément, P. (1975): Approximation by finite element functions using local regularizations. RAIRO Modél. Math. Anal. Numér., 2, 77-84. MR 53:4569

[Dö1] Dörfler, W. (1996): A convergent adaptive algorithm for Poisson's equation. SIAM J. Numer. Anal., 33, 1106-1124. MR 97e:65139

[Dö2] Dörfler, W. (1995): A robust adaptive strategy for the nonlinear Poisson equation. Computing, 55, 289-304. MR 97e:65111

[GH] George, P. L., Hermeline, F. (1992): Delaunay's mesh of a convex polyhedron in dimension d. Internat. J. Numer. Methods Engrg., 33, 975-995. MR 93e:65117

[HTH] Hamann, B., Thornburg, H. J., Hong, G. (1995): Automatic unstructured grid generation based on iterative point insertion. Computing, 55, 135-161. MR 96d:65036 
[Ka] Kadlec, J. (1964): On the regularity of the solution of the Poisson problem on a domain with boundary locally similar to the boundary of a convex open set (in Russian). Czechoslovak Math. J., 14, 386-393. MR 30:329

[LM] Lions, J. L., Magenes, E. (1972): Non-homogeneous boundary value problems and applications I., Springer, New York. MR 50:2670

[Re] Rebay, S. (1993): Efficient unstructured mesh generation by means of Delaunay triangulation and Bowyer-Watson algorithm. J. Comput. Physics, 106, 125-138.

[Ri] Rivara, M.-C. (1984): Algorithms for refining triangular grids suitable for adaptive and multigrid techniques. Internat. J. Numer. Methods Engrg., 20, 745-756. MR 85h:65258

[SZ] Scott, L. R., Zhang, S. (1990): Finite element interpolation of nonsmooth functions satisfying boundary conditions, Math. Comp., 54, 483-493 MR 90j:65021

[SM] Spanier, J., Maize, E. H (1994): Quasi-random methods for estimating integrals using relatively small samples. SIAM Review, 36, 18-44. MR 95b:65013

[Ve1] Verfürth, R. (1994): A posteriori error estimation and adaptive mesh-refinement techniques. J. Comput. Appl. Math., 50, 67-83. MR 95c:65171

[Ve2] Verfürth, R. (1994): A posteriori error estimates for nonlinear problems. Math. Comp., 62, 445-475. MR 94j:65136

[We] Weatherhill, N. P. (1992): Delaunay triangulation in CFD. Comput. Math. Appl., 24.2, 129-150.

[Y] Yserentant, H. (1986): On the multi-level splitting of finite element spaces, Numer. Math. 49 (1986), 379-412. MR 88d:65068a

Institut für Angewandte Mathematik, Universität Freiburg, Hermann-HerderStrasse 10, D-79104 Freiburg, Germany

E-mail address: willy@mathematik.uni-freiburg.de

Institut für Angewandte Mathematik, Universität Bonn, Wegelerstrasse 6, D-52115 Bonn, Germany

E-mail address: rumpf@iam.uni-bonn.de 